\title{
Twisted particle collisions: A new tool for spin physics
}

\author{
Igor P. Ivanov $\odot,{ }^{1, *}$ Nikolai Korchagin, ${ }^{2, \dagger}$ Alexandr Pimikov $\odot,{ }^{2,3, \hbar}$ and Pengming Zhang $\oplus^{4, \S}$ \\ ${ }^{1}$ CFTP, Instituto Superior Tecnico, Universidade de Lisboa, Lisbon 1049-001, Portugal \\ ${ }^{2}$ Institute of Modern Physics, Chinese Academy of Sciences, Lanzhou 730000, China \\ ${ }^{3}$ Research Institute of Physics, Southern Federal University, Rostov-na-Donu 344090, Russia \\ ${ }^{4}$ School of Physics and Astronomy, Sun Yat-sen University, Zhuhai 519082, China
}

(Received 6 March 2020; accepted 2 May 2020; published 15 May 2020)

\begin{abstract}
Collisions of twisted particles - that is, non-plane-wave states of photons, electrons, or any other particle, equipped with a nonzero orbital angular momentum (OAM) with respect to its propagation direction-offer novel ways to probe particle structure and interactions. Recently, we argued that resonance production in twisted photon collisions or twisted $e^{+} e^{-}$annihilation gives access to parity- and spinsensitive observables in inclusive cross sections, even when the initial particles are unpolarized. Here, we explore these features in detail, providing a qualitative picture and illustrating it with numerical examples. We show how one can detect parity-violating effects in collisions of unpolarized twisted photons and how one can produce almost $100 \%$ polarized vector mesons in unpolarized twisted $e^{+} e^{-}$annihilation. These examples highlight the unprecedented level of control over polarization offered by twisted particles, impossible in the usual plane wave collisions.
\end{abstract}

DOI: 10.1103/PhysRevD.101.096010

\section{INTRODUCTION}

Determining the spin and parity properties of hadrons is an intricate and fascinating aspect of modern particle phenomenology. Known hadrons, including the very short-lived resonances, exhibit a variety of spin-parity quantum numbers possible with the usual $q \bar{q}$ and $q q q$ quark combinations $[1,2]$, with multiquark states [3,4] and glueball states [5]. Deep inelastic scattering (DIS) with a polarized initial lepton or proton allows one to investigate how spin of the ultrarelativistic proton emerges from spins and orbital angular momenta of its constituents [6]. This problem alone, dubbed the spin proton crisis, has remained a source of controversies over the past decades, and the situation is still far from being completely resolved [7]. Going beyond helicity distributions and entering the realm of the three-dimensional (3D) momentum space spin structure brings in many new spin-sensitive variables, which can be encoded via transverse-momentum distributions and explored experimentally in semi-inclusive DIS with transversely polarized protons $[8,9]$. The recently

\footnotetext{
*igor.ivanov@tecnico.ulisboa.pt

tkorchagin@impcas.ac.cn

pimikov@mail.ru

zhangpm5@mail.sysu.edu.cn
}

Published by the American Physical Society under the terms of the Creative Commons Attribution 4.0 International license. Further distribution of this work must maintain attribution to the author(s) and the published article's title, journal citation, and DOI. Funded by SCOAP ${ }^{3}$. approved Electron Ion Collider in the United States (EIC) [10] and the proposed Electron Ion Collider in China (EicC) [11] also have a rich spin physics program ahead.

There are two main experimental tools for investigating spin-parity properties of hadrons. First, one can produce them in collision of longitudinally or transversely polarized initial particles and measure spin asymmetries, that is, the response of the cross section to flipping the polarization sign or changing the polarization direction. Second, one can study exclusive or semi-inclusive reactions; look into angular distribution of the final state particles; and, with the aid of partial-wave analysis, deduce the spin properties either of the target hadron (in DIS) or of the intermediate resonances (in low-energy exclusive production processes). In either case, the task requires certain experimental efforts in preparing a polarized initial state or in extracting the angular correlations of the final state particles. There seems to be no other way to access spin-dependent observables.

In the recent paper [12], we proposed a completely new tool for doing spin physics in particle collisions. We showed that if one prepares initial particles in the so-called twisted state, in which they are equipped with a nonzero, adjustable orbital angular momentum (OAM) with respect to their propagation direction, then spin- and parity-dependent observables can be probed with fully inclusive cross sections of unpolarized particles. In this paper, we provide a detailed exploration of this idea by considering production of spin- 0 or spin- 2 resonances in twisted $\gamma \gamma$ collisions and of spin-1 resonance in twisted $e^{+} e^{-}$annihilation. 
We present a qualitative picture, which helps us understand several consequences which otherwise may seem counterintuitive, and corroborate it with numerical examples.

In the following section, we give a brief reminder of how twisted photons and electrons are described and equip the reader with expressions for calculations of the twisted helicity amplitudes. We also discuss in detail the subtle notion of unpolarized twisted photon or electron beam. Then, in Sec. III, we calculate production of spin-0 and spin-2 resonances in twisted photon collisions. We show how one can extract scalar-pseudoscalar mixing in spin-0 production and how to selectively produce a spin-2 particle in a specific polarization state-all done with unpolarized twisted photon beams. In Sec. IV, we repeat the analysis for vector meson production in unpolarized twisted $e^{+} e^{-}$ annihilation. We finish the paper with an outline of the experimental prospects to study the effects propose and a summary of our results.

Throughout the paper, we use natural units $\hbar=c=1$. Three-dimensional vectors will be denoted by bold symbols, while the transverse momenta will be labeled by the subscript $\perp$. We will often use the shorthand notations $c$ and $s$, which stand for

$c \equiv \cos \theta, \quad s \equiv \sin \theta$ for scalar and vector fields;

$c \equiv \cos (\theta / 2), \quad s \equiv \sin (\theta / 2)$ for electrons and positrons.

\section{DESCRIBING TWISTED PARTICLES}

\section{A. Twisted scalar particles}

Description of twisted particle states in a way convenient for calculations of high-energy collision processes was first presented in Refs. [13,14] by adapting the most general framework of Ref. [15]. It was further developed in Refs. [16-23]; see also the recent reviews on twisted electrons [24,25] and photons [26,27]. In this section, we will recapitulate this formalism, first for scalar twisted states and then for photons and electrons. The calculations become most transparent for the so-called Bessel twisted states described below. This is not the only option available. Collisions involving Laguerre-Gaussian twisted states $[21,23]$ and other wave packets with nontrivial phase structure [20] also demonstrated remarkable features not present in (approximate) plane wave collisions. However, for the purposes of the present paper, we find it sufficient to stay with (Gaussian-smeared) Bessel states.

A Bessel twisted state is a solution of the free wave equation with a definite energy $E$, longitudinal momentum $k_{z}$, modulus of the transverse momentum $\left|\mathbf{k}_{\perp}\right|=\varkappa$, and a definite $z$ projection of the total angular momentum $m$, which must be integer. Since, for the scalar field, the total angular momentum coincides with the OAM, the same parameter $m$ also quantifies the $z$ projection of the OAM. Written in cylindric coordinates $\rho, \varphi_{r}, z$, this solution $|E, \varkappa, m\rangle$ has the form

$$
\begin{aligned}
& |E, \varkappa, m\rangle=e^{-i E t+i k_{z} z} \cdot \psi_{\varkappa m}\left(\mathbf{r}_{\perp}\right), \\
& \psi_{\varkappa m}\left(\mathbf{r}_{\perp}\right)=e^{i m \varphi_{r}} \sqrt{\frac{\varkappa}{2 \pi}} J_{m}(\varkappa \rho),
\end{aligned}
$$

where $J_{m}(x)$ is the Bessel function. This function is normalized according to

$$
\int d^{2} \mathbf{r}_{\perp} \psi_{\varkappa^{\prime} m^{\prime}}^{*}\left(\mathbf{r}_{\perp}\right) \psi_{\varkappa m}\left(\mathbf{r}_{\perp}\right)=\delta\left(\varkappa-\varkappa^{\prime}\right) \delta_{m, m^{\prime}}
$$

The azimuthal angle dependence $\propto e^{i m \varphi_{r}}$ is the hallmark feature of the phase vortex. A twisted state can be represented as a superposition of plane waves,

$$
|E, \varkappa, m\rangle=e^{-i E t+i k_{z} z} \int \frac{d^{2} \mathbf{k}_{\perp}}{(2 \pi)^{2}} a_{\varkappa m}\left(\mathbf{k}_{\perp}\right) e^{i \mathbf{k}_{\perp} \mathbf{r}_{\perp}}
$$

where

$$
a_{\varkappa m}\left(\mathbf{k}_{\perp}\right)=(-i)^{m} e^{i m \varphi_{k}} \sqrt{\frac{2 \pi}{\varkappa}} \delta\left(\left|\mathbf{k}_{\perp}\right|-\varkappa\right) .
$$

is the corresponding Fourier amplitude. This expansion can be inverted, which means that twisted states form a complete basis for (transverse) wave functions $[13,14]$.

When passing from plane waves to twisted states, one should also take care of the change of the normalization factors. The accurate treatment of these factors can be found in Refs. $[18,28]$. Here, although, the appropriate normalization coefficients are implicitly assumed, and we do not write them for the following reason. The absolute value of the twisted scattering cross section depends not only on the dynamics of the fundamental interactions but also on the details of how the initial twisted state is prepared. These details depend on the eventual experimental realization of the twisted states. Therefore, in contrast to the usual plane wave setting, the absolute value of the cross section cannot be unambiguously predicted.

If one looks into the integrated cross section, its departure from the plane wave cross section is typically small and often negligible; see the very recent study [23]. However, the most dramatic novelties of the two-twistedparticle collision arise not in the absolute value of the cross section but in differential distributions absent in the plane wave case. Since the absolute value of the cross section is not the figure of merit for the present study, we will often skip the normalization factors and plot cross sections in arbitrary units.

We remark in passing that sometimes a different normalization of $a_{x m}\left(\mathbf{k}_{\perp}\right)$ is adopted, namely, with the coefficient $2 \pi / \varkappa$ instead of $\sqrt{2 \pi / \varkappa}$. This is the consequence of a different normalization condition for the coordinate wave function: with or without the prefactor $2 \pi / \varkappa$ in Eq. (2). This difference does not change the 
observables; one just needs to keep track of the exact normalization choice when calculating the event rate and the flux.

If the above Bessel state describes a particle with mass $\mu$, the energy and momentum are related as $E^{2}=\mu^{2}+\varkappa^{2}+k_{z}^{2}$. Notice that the average momentum of this state $\langle\mathbf{k}\rangle=$ $\left(0,0, k_{z}\right)$ does not satisfy the dispersion relation: $E^{2}=$ $\mu^{2}+\varkappa^{2}+\langle\mathbf{k}\rangle^{2} \neq \mu^{2}+\langle\mathbf{k}\rangle^{2}$. Whether to interpret the quantity $\mu^{2}+\varkappa^{2}$ as a new "effective mass" squared is just a matter of terminological convenience.

Just like a plane wave, a pure Bessel state $|E, \varkappa, m\rangle$ with fixed $\varkappa$ is non-normalizable in the transverse plane. Although the resulting singularities can be dealt with $[13,14,16,18]$, it is more appropriate to use realistic, transversely localized monochromatic beams. ${ }^{1}$ Such a beam can be written as a superposition of Bessel states with equal energy and equal values of $m$ but with a distribution over $\varkappa$,

$$
|E, \bar{\varkappa}, \sigma, m\rangle=\int d \varkappa f(\varkappa)|E, \varkappa, m\rangle .
$$

The weight function $f(\varkappa)$ should be peaked at $\bar{x}$ and have a width $\sigma$; apart from that, it is unconstrained and will depend on details of a future experimental realization scheme. In our calculations below, we will use the Gaussian function corrected by a slow-varying prefactor:

$$
f(\varkappa)=n \sqrt{\varkappa} \exp \left[-\frac{(\varkappa-\bar{\varkappa})^{2}}{2 \sigma^{2}}\right],
$$

with the normalization condition $\int_{0}^{E} d \varkappa|f(\varkappa)|^{2}=1$.

\section{B. Description of twisted photons}

When describing twisted photons, we adapt the formalism of Refs. [13,14,27]. For definiteness, we will work in the Coulomb gauge, where all polarization vectors only have the spatial components. A monochromatic plane wave electromagnetic field with helicity $\lambda= \pm 1$ is described by

$$
\mathbf{A}_{\mathbf{k} \lambda}(\mathbf{r})=\mathbf{e}_{\mathbf{k} \lambda} e^{i \mathbf{k r}} .
$$

The polarization vector is orthogonal to the wave vector: $\mathbf{e}_{\mathbf{k} \lambda} \mathbf{k}=0$. Quantization of this field produces plane wave photons with momentum $\mathbf{k}$.

As for the scalar case, we fix a reference frame, select an axis $z$, and construct a Bessel twisted photon as a superposition of plane waves with fixed longitudinal momentum $k_{z}=|\mathbf{k}| \cos \theta$ and fixed modulus of the

\footnotetext{
${ }^{1}$ We stress that a monochromatic solution with a localized transverse wave function cannot be localized in the $z$ direction; otherwise, monochromaticity is lost. Therefore, such solutions correspond to beams rather than wave packets, although we will occasionally use the latter term as well.
}

transverse momentum $\varkappa=\left|\mathbf{k}_{\perp}\right|=k \sin \theta$, but arriving from different azimuthal angles $\varphi_{k}$. Such a twisted photon with a definite $z$ projection of the total angular momentum $m$ and definite helicity $\lambda= \pm 1$ can be written as

$$
\mathbf{A}_{\varkappa m \lambda}(\mathbf{r})=e^{i k_{z} z} \int a_{\varkappa m}\left(\mathbf{k}_{\perp}\right) \mathbf{e}_{\mathbf{k} \lambda} e^{i \mathbf{k}_{\perp} \mathbf{r}_{\perp}} \frac{d^{2} \mathbf{k}_{\perp}}{(2 \pi)^{2}}
$$

where the Fourier amplitude $a_{\varkappa m}\left(\mathbf{k}_{\perp}\right)$ is given by the same Eq. (4). The usual dispersion relation holds for every plane wave component: $k_{z}^{2}+\varkappa^{2}=E^{2}$.

In contrast to the scalar case, each plane wave component of a twisted photon contains its polarization vector $\mathbf{e}_{\mathbf{k} \lambda}$, which is orthogonal to the momentum of that particular plane wave component: $\mathbf{e}_{\mathbf{k} \lambda} \mathbf{k}=0$. As a result, the polarization vector cannot be taken out of the integral. Back in the coordinate space, the polarization state of a twisted photon is described by a polarization field rather than a polarization vector.

To describe the polarization vector of a photon with an arbitrary momentum, let us define the eigenvectors $\chi_{\sigma}$, $\sigma= \pm 1,0$, of the helicity operator $\hat{s}_{z}$ defined with respect to the axis $z: \hat{s}_{z} \chi_{\sigma}=\sigma \chi_{\sigma}$. Their explicit form is

$$
\chi_{0}=\left(\begin{array}{l}
0 \\
0 \\
1
\end{array}\right), \quad \chi_{ \pm 1}=\frac{\mp 1}{\sqrt{2}}\left(\begin{array}{c}
1 \\
\pm i \\
0
\end{array}\right), \quad \chi_{\sigma}^{*} \chi_{\sigma^{\prime}}=\delta_{\sigma \sigma^{\prime}} .
$$

The polarization vector can be expanded in the basis of $\chi_{\sigma}$ :

$$
\mathbf{e}_{\mathbf{k} \lambda}=\sum_{\sigma=0, \pm 1} e^{-i \sigma \varphi_{k}} d_{\sigma \lambda}^{1}(\theta) \chi_{\sigma}
$$

The explicit expressions for Wigner's $d$ functions [29] are

$$
d_{\sigma \lambda}^{1}=\left(\begin{array}{ccc}
\cos ^{2} \frac{\theta}{2} & -\frac{1}{\sqrt{2}} \sin \theta & \sin ^{2} \frac{\theta}{2} \\
\frac{1}{\sqrt{2}} \sin \theta & \cos \theta & -\frac{1}{\sqrt{2}} \sin \theta \\
\sin ^{2} \frac{\theta}{2} & \frac{1}{\sqrt{2}} \sin \theta & \cos ^{2} \frac{\theta}{2}
\end{array}\right) .
$$

The first, second, and third rows and columns of this matrix correspond to the indices $+1,0$, and -1 . Performing the summation in Eq. (10), one gets explicit expressions for the polarization vectors:

$\mathbf{e}_{\mathbf{k} \lambda}=\frac{\lambda}{\sqrt{2}}\left(\begin{array}{c}-\cos \theta \cos \varphi_{k}+i \lambda \sin \varphi_{k} \\ -\cos \theta \sin \varphi_{k}-i \lambda \cos \varphi_{k} \\ \sin \theta\end{array}\right), \quad \lambda= \pm 1$.

Notice that the Fourier amplitude $a_{x m}\left(\mathbf{k}_{\perp}\right)$ is an eigenstate not only of $\hat{J}_{z}$, the operator of the $z$ component of the total angular momentum, but also of $\hat{L}_{z}=-i \partial / \partial \varphi_{k}$, the $z$ projection of the OAM operator. However, this property is not shared by $\mathbf{e}_{\mathbf{k} \lambda}$ given above; it is an eigenstate of $\hat{J}_{z}$ with 
the zero eigenvalue but not of $\hat{L}_{z}$ or $\hat{s}_{z}$ separately. This polarization vector, even for fixed $\lambda$, contains contributions with different $s_{z}$ and $\ell=L_{z}$, which sum up to zero. Thus, the twisted photon (8), strictly speaking, is not an eigenstate of the OAM because the spin and OAM projections are not conserved separately even for free electromagnetic fields.

In most experimental situations, twisted photons are produced in the paraxial regime, where $\theta \ll 1$. In this case, one can talk about approximately conserved $s_{z}=\lambda$ and $\ell=m-\lambda$. Indeed, when $\theta \rightarrow 0$, the polarization vector becomes

$$
\mathbf{e}_{\mathbf{k} \lambda} \rightarrow e^{-i \lambda \varphi_{k}} \chi_{\lambda},
$$

which now has definite $s_{z}=-\ell=\lambda$. Beyond the paraxial approximation, the spin-orbital interaction, which exists for free electromagnetic waves, comes into play and gives rise to a variety of remarkable optical phenomena [30]. In particular, it leads to spatially varying polarization states of light described by polarization field. In a tightly focused light beam, the polarization field evolves downstream and may significantly differ at the aperture and in the focal plane.

Finally, when describing a counterpropagating twisted photon defined in the same reference frame with respect to the same axis $z$, one can use the above expressions assuming that $k_{z}<0$ and replacing $m \rightarrow-m$ in the Fourier amplitude (4). The expression for the polarization vector (12) stays unchanged, but $\cos \theta<0$. The paraxial limit is now given by $\theta \rightarrow \pi$, in which case $\mathbf{e}_{\mathbf{k} \lambda} \rightarrow e^{+i \lambda \rho_{k}} \chi_{-\lambda}$.

\section{Description of twisted electrons and positrons}

Twisted states have been experimentally demonstrated not only for photons but also for electrons [31-33]. To describe them in a fully relativistic manner, we use the definitions of Refs. [24,28]; other works, such as Refs. [18,34], use slightly different conventions. The plane wave electron with the four-momentum $k^{\mu}=\left(E, \mathbf{k}_{\perp}, k_{z}\right)$, corresponding to the propagation direction with angles $\theta$ and $\varphi_{k}$ and with helicity $\zeta= \pm 1 / 2$ is described by

$$
\Psi_{k \zeta}(\mathbf{r})=\frac{1}{\sqrt{2 E}} u_{\zeta}(k) e^{i \mathbf{k r}}
$$

The bispinor $u_{\zeta}(k)$ used here is

$$
\begin{aligned}
u_{\zeta}(k) & =\left(\begin{array}{c}
\sqrt{E+m_{e}} w^{(\zeta)} \\
2 \zeta \sqrt{E-m_{e}} w^{(\zeta)}
\end{array}\right), \quad w^{(+1 / 2)}=\left(\begin{array}{c}
c e^{-i \varphi_{k} / 2} \\
s e^{i \varphi_{k} / 2}
\end{array}\right), \\
w^{(-1 / 2)} & =\left(\begin{array}{c}
-s e^{-i \varphi_{k} / 2} \\
c e^{i \varphi_{k} / 2}
\end{array}\right),
\end{aligned}
$$

where $c \equiv \cos (\theta / 2), \quad s \equiv \sin (\theta / 2)$. The bispinors are normalized as $\bar{u}_{\zeta_{1}}(k) u_{\zeta_{2}}(k)=2 m_{e} \delta_{\zeta_{1}, \zeta_{2}}$. The negativefrequency solutions of the Dirac equation are constructed as

$$
v_{\zeta}(k)=\left(\begin{array}{c}
-\sqrt{E-m_{e}} w^{(-\zeta)} \\
2 \zeta \sqrt{E+m_{e}} w^{(-\zeta)}
\end{array}\right)
$$

with the same spinors $w$ as in (15). We use this basis of plane wave solutions of the Dirac equation to construct the Bessel vortex state of the electron,

$$
\Psi_{\varkappa m k_{z} \zeta}(\mathbf{r})=e^{i k_{z} z} \int a_{\varkappa m}\left(\mathbf{k}_{\perp}\right) \frac{u_{\zeta}(k)}{\sqrt{2 E}} e^{i \mathbf{k}_{\perp} \mathbf{r}_{\perp}} \frac{d^{2} \mathbf{k}_{\perp}}{(2 \pi)^{2}},
$$

with the same Fourier amplitude $a_{\varkappa m}\left(\mathbf{k}_{\perp}\right)$ as in Eq. (4). Notice that the total angular momentum projection $m$ is now half-integer. The similar expression holds for the negative-frequency solutions.

Just as in the case of twisted photons, the spin and orbital angular momentum projections are not separately conserved due to the intrinsic spin-orbital interaction of the twisted electron [21,34]. In the paraxial approximation, when the spin-orbital interaction is suppressed, one can nevertheless talk about two approximately conserved quantum numbers: the $z$ projection of the spin operator with $s_{z}=\zeta$ and the $z$ projection of the OAM with $\ell=m-\zeta$. One could also define Bessel electron states in which the spinor $u_{k \zeta}$ contains an extra factor $\exp \left(i \zeta \varphi_{k}\right)$, while the Fourier amplitude (4) is constructed with integer $\ell$ instead of half-integer $m$ [18]. This is also a valid Bessel electron solution; its total angular momentum depends on helicity, $m=\ell+\zeta$, while the parameter $\ell$ characterizes the orbital angular momentum independent of helicity. These two conventions correspond to two definitions of how an unpolarized electron is defined; see below.

\section{Unpolarized twisted photons or electrons}

Let us discuss the subtle notion of unpolarized twisted photons. For concreteness, we talk about photons, but the entire discussion is applicable to electrons and other particles with spin.

Because of the presence of spin-orbital interaction of light in free space, the notion of unpolarized twisted light is not unambiguously defined. For a plane-wave photon, with its polarization vector independent of spatial coordinates, one can think of unpolarized light as an equal mixture of photons in two orthogonal polarization states, for examples, with $\lambda=+1$ and $\lambda=-1$. For twisted light, an ambiguity arises: when considering photons with $\lambda= \pm 1$, should we keep the total angular momentum $m$ fixed? Or should we fix $m-\lambda$, which would correspond in the paraxial limit to the same spatial distribution of the two polarization states?

There is no unique answer to this question; it will depend on the photon preparation details in every experimental scheme. If an experimental device manages to select photons with a single $m$ irrespective of the photon helicity, then one needs to calculate the process of interest (in our case, the cross section) with $|m, \lambda=+1\rangle$ and $|m, \lambda=-1\rangle$ and perform the averaging. If one creates twisted photons 
by letting them pass through a fixed aperture plate which would impose a given OAM $\ell$ in the scalar case, then, immediately behind the aperture, one can reliably describe the unpolarized twisted light as consisting of photons with $\left|m_{+}=\ell+1, \lambda=+1\right\rangle$ and $\left|m_{-}=\ell-1, \lambda=-1\right\rangle$. However, this description evolves downstream and may become very different in the focal spot due to the same spinorbital interaction of light. An example of this evolution known as spin-to-orbital conversion was experimentally demonstrated in Ref. [35]. In that work, circularly polarized light passed through a specially designed numerical aperture, acquired OAM, and drove rotation of an ensemble of microscopic target particles in the focal plane. Although the left and right circularly polarized light had the same spatial distribution before the aperture, this distribution evolved in a different manner on the way to the focal plane and led to different rotation patterns of the ensemble.

It means that when calculating processes with unpolarized twisted photons in realistic settings one must specify according to which definition the twisted light is unpolarized. It is very possible that the realistic situation will correspond to an intermediate definition between the two options just described. In the next section, when describing twisted photon collisions, we will discuss how the results differ with the two definitions of unpolarized twisted photons: fixed- $m$ and fixed$\ell$ options. We will show that with both definitions the key spin- or parity-sensitive observables do not vanish, although their magnitude will be different. Their value in a realistic experimental situation will likely lie in between.

\section{RESONANCE PRODUCTION IN TWISTED PHOTON COLLISIONS}

\section{A. General features of twisted particle annihilation}

Let us begin by briefly recapitulating the main features of two twisted particles collisions; see more details in Ref. [36].

If we were to describe a $2 \rightarrow 1$ annihilation process for the plane wave case, we would need to write the $S$-matrix amplitude as

$$
\begin{aligned}
S_{P W}= & i(2 \pi)^{4} \delta\left(E_{1}+E_{2}-E_{K}\right) \\
& \times \delta^{(3)}\left(\mathbf{k}_{1}+\mathbf{k}_{2}-\mathbf{K}\right) \frac{\mathcal{M}\left(k_{1}, k_{2} ; K\right)}{\sqrt{8 E_{1} E_{2} E_{K}}} .
\end{aligned}
$$

Here, the energies and momenta of the initial particles are $E_{i}$ and $\mathbf{k}_{i}$, for the final particles $E_{K}$ and $\mathbf{K} . \mathcal{M}\left(k_{1}, k_{2} ; K\right)$ is the invariant amplitude calculated according to the standard Feynman rules. Squaring this amplitude and appropriately regularizing the squares of delta functions, as described, for instance, in Ref. [37], we would get the cross section

$$
\begin{aligned}
d \sigma & =\frac{\pi \delta\left(E_{1}+E_{2}-E_{K}\right)}{4 E_{1} E_{2} E_{K} v}|\mathcal{M}|^{2} \delta^{(3)}\left(\mathbf{k}_{1}+\mathbf{k}_{2}-\mathbf{K}\right) d^{3} K \\
\sigma & =\frac{\pi \delta\left(E_{1}+E_{2}-E_{K}\right)}{4 E_{1} E_{2} E_{K} v}|\mathcal{M}|^{2}
\end{aligned}
$$

Notice the well-known features of this cross section: the final momentum is fixed at $\mathbf{K}=\mathbf{k}_{1}+\mathbf{k}_{2}$, and the dependence on the total energy of the colliding particles is proportional to $\delta\left(E_{1}+E_{2}-E_{K}\right)$. The production process occurs only when the initial particles are directly "on resonance."

Let us now consider collision of two Bessel states $\left|E_{1}, \varkappa_{1}, m_{1}\right\rangle$ and $\left|E_{2}, \varkappa_{2}, m_{2}\right\rangle$, which are defined with respect to the same axis $z$. The final particle with mass $M$ is still described in the basis of plane waves with the momentum $\mathbf{K}$ and energy $E_{K}$. The $S$-matrix element of this process is

$$
\begin{aligned}
S & =\int \frac{d^{2} \mathbf{k}_{1 \perp}}{(2 \pi)^{2}} \frac{d^{2} \mathbf{k}_{2 \perp}}{(2 \pi)^{2}} a_{\varkappa_{1} m_{1}}\left(\mathbf{k}_{1 \perp}\right) a_{\varkappa_{2},-m_{2}}\left(\mathbf{k}_{2 \perp}\right) S_{P W} \\
& =i(2 \pi)^{4} \frac{\delta(\Sigma E) \delta\left(\Sigma k_{z}\right)}{\sqrt{8 E_{1} E_{2} E_{K}}} \frac{(-i)^{m_{1}-m_{2}}}{(2 \pi)^{3} \sqrt{\varkappa_{1} \varkappa_{2}}} \cdot \mathcal{J},
\end{aligned}
$$

where $\delta(\Sigma E) \equiv \delta\left(E_{1}+E_{2}-E_{K}\right), \delta\left(\Sigma k_{z}\right) \equiv \delta\left(k_{1 z}+k_{2 z}-K_{z}\right)$. The twisted amplitude $\mathcal{J}$ is defined as

$$
\begin{aligned}
\mathcal{J}= & \int d^{2} \mathbf{k}_{1 \perp} d^{2} \mathbf{k}_{2 \perp} e^{i m_{1} \varphi_{1}-i m_{2} \varphi_{2}} \delta\left(\left|\mathbf{k}_{1 \perp}\right|-\varkappa_{1}\right) \\
& \times \delta\left(\left|\mathbf{k}_{2 \perp}\right|-\varkappa_{2}\right) \delta^{(2)}\left(\mathbf{k}_{1 \perp}+\mathbf{k}_{2 \perp}-\mathbf{K}_{\perp}\right) \cdot \mathcal{M} \\
& =\varkappa_{1} \varkappa_{2} \int d \varphi_{1} d \varphi_{2} e^{i m_{1} \varphi_{1}-i m_{2} \varphi_{2}} \delta^{(2)}\left(\mathbf{k}_{1 \perp}+\mathbf{k}_{2 \perp}-\mathbf{K}_{\perp}\right) \cdot \mathcal{M} .
\end{aligned}
$$

Remarkably, the twisted amplitude $\mathcal{J}$ can be calculated exactly [16]. It is nonzero only if $\varkappa_{i}$ and $K \equiv\left|\mathbf{K}_{\perp}\right|$ satisfy the triangle inequalities

$$
\left|\varkappa_{1}-\varkappa_{2}\right| \leq K \leq \varkappa_{1}+\varkappa_{2} .
$$

They form a triangle with the area

$\Delta=\frac{1}{4} \sqrt{2 K^{2} \varkappa_{1}^{2}+2 K^{2} \varkappa_{2}^{2}+2 \varkappa_{1}^{2} \varkappa_{2}^{2}-K^{4}-\varkappa_{1}^{4}-\varkappa_{2}^{4}}$.

Out of many plane wave components "stored" in the initial twisted particles, the integral (21) receives contributions from exactly two plane wave combinations shown in Fig. 1 with the following azimuthal angles:
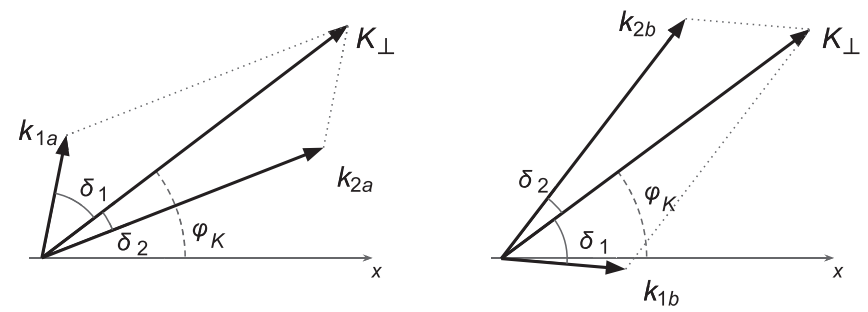

FIG. 1. The two kinematical configurations in the transverse plane that satisfy momentum conservation laws in the scattering of two Bessel electron states. 
configuration a: $\varphi_{1}=\varphi_{K}+\delta_{1}, \quad \varphi_{2}=\varphi_{K}-\delta_{2}$, configuration b: $\varphi_{1}=\varphi_{K}-\delta_{1}, \quad \varphi_{2}=\varphi_{K}+\delta_{2}$.

Notice that

$$
\begin{aligned}
& \delta_{1}=\arccos \left(\frac{\varkappa_{1}^{2}+K^{2}-\varkappa_{2}^{2}}{2 \varkappa_{1} K}\right), \\
& \delta_{2}=\arccos \left(\frac{\varkappa_{2}^{2}+K^{2}-\varkappa_{1}^{2}}{2 \varkappa_{2} K}\right)
\end{aligned}
$$

are the inner angles of the triangle with the sides $\varkappa_{1}, \varkappa_{2}, K$; they are not azimuthal variables.

The result for the twisted amplitude $\mathcal{J}$ can then be compactly written as

$\mathcal{J}=e^{i\left(m_{1}-m_{2}\right) \varphi_{K}} \frac{\varkappa_{1} \varkappa_{2}}{2 \Delta}\left[\mathcal{M}_{a} e^{i\left(m_{1} \delta_{1}+m_{2} \delta_{2}\right)}+\mathcal{M}_{b} e^{-i\left(m_{1} \delta_{1}+m_{2} \delta_{2}\right)}\right]$.

Notice that the plane wave amplitudes $\mathcal{M}_{a}$ and $\mathcal{M}_{b}$ are calculated for the two distinct initial momentum configurations shown in Fig. 1 but for the same final momentum $\mathbf{K}$. They exhibit two distinct paths in momentum space to arrive at the same final state from the initial twisted states. In a sense, scattering of twisted Bessel states represents the momentum-space analog of the Young double-slit experiment [38].

Squaring (20) and performing appropriate regularization, we obtain the (generalized) cross section in the form

$$
d \sigma \propto|\mathcal{J}|^{2} \delta\left(E_{1}+E_{2}-E_{K}\right) d^{2} \mathbf{K}_{\perp} .
$$

We deliberately omitted the prefactor to stress, as we discussed in the previous section, that the absolute value of the cross section cannot be predicted unambiguously as it depends on the details of initial state preparation and, therefore, it is not the figure of merit. Instead, it is the nontrivial distribution over $\mathbf{K}_{\perp}$, which was absent in the plane wave case (19), that we pay attention to.

For fixed initial values of $E_{i}, \varkappa_{i}$, and $M$, the energymomentum conservation fixes $K_{z}=k_{1 z}+k_{2 z}$ and, therefore, the modulus of the transverse momentum $K=$ $\sqrt{E_{K}^{2}-M^{2}-K_{z}^{2}}$. Thus, the polar angle of the produced resonance is fixed [36],

$$
\cos \theta_{K}=\frac{K_{z}}{\sqrt{\left(E_{1}+E_{2}\right)^{2}-M^{2}}},
$$

but the cross section exhibits a uniform distribution in the azimuthal angle.

The expression for the cross section (27) and the exact evaluation of $\mathcal{J}$ in (26) were obtained for the pure Bessel states, which are not normalizable and lead to singularities in the cross sections. These singularities are removed for realistic twisted wave packets with a finite transverse extent, for which we use the monochromatic Gaussiansmeared wave packet given in Eqs. (5) and (6). This smearing with the functions $f_{1}\left(\varkappa_{1}\right)$ and $f_{2}\left(\varkappa_{2}\right)$ must be applied at the level of $S$-matrix amplitude (20). Therefore, instead of pure Bessel twisted amplitude $\mathcal{J}$, we evaluate its smeared counterpart:

$$
\langle\mathcal{J}\rangle=\int d \varkappa_{1} d \varkappa_{2} f_{1}\left(\varkappa_{1}\right) f_{2}\left(\varkappa_{2}\right) \delta\left(k_{1 z}+k_{2 z}-K_{z}\right) \frac{\mathcal{J}}{\sqrt{\varkappa_{1} \varkappa_{2}}} .
$$

Notice that this integration now affects the longitudinal momenta, since, for monoenergetic states, variation of $\varkappa$ induces variation of $k_{z}$. Therefore, the final particle now displays a 2D momentum space distribution, which can be written as

$$
d \sigma \propto E_{K}^{2} \beta_{K}|\langle\mathcal{J}\rangle|^{2} d \Omega_{K}=\frac{E_{K}}{K_{z}}|\langle\mathcal{J}\rangle|^{2} d^{2} \mathbf{K}_{\perp} .
$$

Further insights into this distribution can be found in Ref. [36].

\section{B. Scalar resonance production in twisted $\gamma \gamma$ collisions}

\section{Exact expressions}

Production of a spin- 0 resonance in twisted $\gamma \gamma$ collision can be described with the same formalism as in the scalar case, corrected for the presence of polarization vectors [39]. One encounters the same twisted amplitude $\mathcal{J}$ as in (21) and (26), where $m_{i}$ now refer to the total angular momentum of each photon, while the invariant amplitude $\mathcal{M}$ depends now on the photon helicities. To calculate it, suppose $S$ is a real scalar field which can be produced in $\gamma \gamma$ collision through the following effective interaction Lagrangian:

$$
\mathcal{L}_{S}=\frac{g}{4} F^{\mu \nu} F_{\mu \nu} S
$$

It generates the helicity amplitude

$$
\mathcal{M}_{S}=g\left[\left(k_{1} k_{2}\right)\left(e_{1} e_{2}\right)-\left(k_{1} e_{2}\right)\left(k_{2} e_{1}\right)\right],
$$

where all products are understood as 4-vector products. For plane wave collisions, one usually chooses the center of motion frame, in which the polarization vectors, written in the Coulomb gauge, are orthogonal to the momenta of both photons, which allows one to drop the second term in (32). In our case, this orthogonality does not hold in the Coulomb gauge, and both terms must be evaluated in the reference frame we work in. 
Using the explicit expressions for the polarization vectors and momenta, one can evaluate the products entering this expression,

$$
\begin{aligned}
\left(k_{1} k_{2}\right)= & \frac{1}{2} M^{2}=E_{1} E_{2}\left[1-c_{1} c_{2}-s_{1} s_{2} \cos \left(\varphi_{1}-\varphi_{2}\right)\right], \\
\left(e_{1} e_{2}\right)= & e^{i\left(\varphi_{1}-\varphi_{2}\right)} \frac{1-\lambda_{1} c_{1}}{2} \frac{1+\lambda_{2} c_{2}}{2} \\
& +e^{-i\left(\varphi_{1}-\varphi_{2}\right)} \frac{1+\lambda_{1} c_{1}}{2} \frac{1-\lambda_{2} c_{2}}{2}-\frac{\lambda_{1} \lambda_{2} s_{1} s_{2}}{2}, \\
\left(e_{1} k_{2}\right)= & \frac{E_{2}}{\sqrt{2}}\left[e^{i\left(\varphi_{1}-\varphi_{2}\right)} \frac{\lambda_{1} c_{1}-1}{2} s_{2}\right. \\
& \left.+e^{-i\left(\varphi_{1}-\varphi_{2}\right)} \frac{1+\lambda_{1} c_{1}}{2} s_{2}-\lambda_{1} s_{1} c_{2}\right],
\end{aligned}
$$

and similarly for $\left(e_{2} k_{1}\right)$. We adopted here the shorthand notation $c_{i} \equiv \cos \theta_{i}, s_{i} \equiv \sin \theta_{i}$. Notice that the plane wave amplitude depends on the azimuthal angles of the two photons only through their difference: $\mathcal{M}_{S}\left(\varphi_{1}, \varphi_{2}\right)=$ $\mathcal{M}_{S}\left(\varphi_{1}-\varphi_{2}\right)$. Substituting these products into (32) and simplifying the expressions, we get a nonzero amplitude only for equal helicities $\lambda_{1}=\lambda_{2}=\lambda$ :

$$
\begin{aligned}
\mathcal{M}_{S}= & 2 g E_{1} E_{2} \delta_{\lambda_{1}, \lambda_{2}}\left[e^{i\left(\varphi_{1}-\varphi_{2}\right)} \frac{1-\lambda c_{1}}{2} \frac{1+\lambda c_{2}}{2}\right. \\
& \left.+e^{-i\left(\varphi_{1}-\varphi_{2}\right)} \frac{1+\lambda c_{1}}{2} \frac{1-\lambda c_{2}}{2}-\frac{s_{1} s_{2}}{2}\right] \\
= & 2 g E_{1} E_{2} \delta_{\lambda_{1}, \lambda_{2}}\left(e_{1}^{(\lambda)} e_{2}^{(\lambda)}\right) .
\end{aligned}
$$

Next, one calculates $\mathcal{J}$ via (26). The two interfering configurations differ only by their azimuthal angles: $\varphi_{1}-\varphi_{2}=\delta_{1}+\delta_{2}$ or $-\left(\delta_{1}+\delta_{2}\right)$. Thus, we get

$$
\begin{aligned}
\mathcal{J}_{S}= & e^{i\left(m_{1}-m_{2}\right) \varphi_{K}} \frac{\varkappa_{1} \varkappa_{2}}{2 \Delta} \cdot g E_{1} E_{2} \delta_{\lambda_{1}, \lambda_{2}} \\
& \times\left\{\left(1-\lambda c_{1}\right)\left(1+\lambda c_{2}\right) \cos \left[m_{1} \delta_{1}+m_{2} \delta_{2}+\delta_{1}+\delta_{2}\right]\right. \\
& +\left(1+\lambda c_{1}\right)\left(1-\lambda c_{2}\right) \cos \left[m_{1} \delta_{1}+m_{2} \delta_{2}-\left(\delta_{1}+\delta_{2}\right)\right] \\
& \left.-2 s_{1} s_{2} \cos \left[m_{1} \delta_{1}+m_{2} \delta_{2}\right]\right\} .
\end{aligned}
$$

In the paraxial limit, when $\theta_{1} \rightarrow 0$ meaning $c_{1} \rightarrow 1$ and $\theta_{2} \rightarrow \pi$ meaning $c_{2} \rightarrow-1$, the first term dominates for $\lambda=-1$, while the second term dominates for $\lambda=+1$. The azimuthal angle dependence $\exp \left[i\left(m_{1}-m_{2}\right) \varphi_{K}\right]$ indicates that the total angular momentum of the initial two-photon system is converted in the OAM of the single final scalar particle, should we want to describe the latter in the basis of twisted states as well [39].

\section{Helicity dependence: Fixed-m case}

A remarkable feature of $\mathcal{J}_{S}$ seen in Eq. (35) is its nontrivial dependence on helicity $\lambda=\lambda_{1}=\lambda_{2}$. Following the discussion in Sec. II D, we now specify that, when considering unpolarized cross section, we fix $m_{1}$ and $m_{2}$ and vary $\lambda= \pm 1$. Then, the expression $\mathcal{J}_{S}$ can be written as

$$
\mathcal{J}_{S}=e^{i\left(m_{1}-m_{2}\right) \varphi_{K}} \delta_{\lambda_{1}, \lambda_{2}} \frac{g E_{1} E_{2} \varkappa_{1} \varkappa_{2}}{\Delta} \cdot\left(\mathcal{J}_{1}+\lambda \mathcal{J}_{2}\right),
$$

where the real quantities $\mathcal{J}_{1}$ and $\mathcal{J}_{2}$ are

$\mathcal{J}_{1}=\cos \left(m_{1} \delta_{1}+m_{2} \delta_{2}\right)\left[\cos \left(\delta_{1}+\delta_{2}\right)\left(1-c_{1} c_{2}\right)-s_{1} s_{2}\right]$,

$\mathcal{J}_{2}=\sin \left(m_{1} \delta_{1}+m_{2} \delta_{2}\right) \sin \left(\delta_{1}+\delta_{2}\right)\left(c_{1}-c_{2}\right)$.

This dependence survives in the cross section, which can be generically represented as

$$
\sigma_{\lambda}=\sigma_{0}+\lambda \sigma_{a} \equiv \sigma_{0}(1+\lambda A),
$$

with $\sigma_{0}$ representing the unpolarized cross section and $\sigma_{a}$ denoting the spin asymmetry. The quantity $A \equiv \sigma_{a} / \sigma_{0}$ can be called the asymmetry contrast. For the pure Bessel beams, this asymmetry contrast is given by

$$
A=\frac{2 \mathcal{J}_{1} \mathcal{J}_{2}}{\mathcal{J}_{1}^{2}+\mathcal{J}_{2}^{2}},
$$

which can vary between -1 and +1 depending on the exact position on the interference fringe.

This dependence may at first look surprising. Indeed, in the familiar plane wave collision, the helicity combinations $\lambda_{1}=\lambda_{2}=+1$ and $\lambda_{1}=\lambda_{2}=-1$ lead to identical cross sections. This is due to the fact the entire process is parity invariant. However, in the present case, we explicitly break the left-right symmetry of the initial state by selecting twisted photons with definite values of $m_{1}$ and $m_{2}$. A different pair of photons with angular momenta $-m_{1}$ and $-m_{2}$ would flip the sign of $\mathcal{J}_{2}$ in (37) and, consequently, in the asymmetry (39). In short, production of a scalar particle in twisted photon collision is invariant under the simultaneous sign flips $m_{i} \rightarrow-m_{i}$ and $\lambda_{i} \rightarrow-\lambda_{i}$, but not under $\lambda_{i} \rightarrow-\lambda_{i}$ alone.

We stress that, for fixed $m$, the asymmetry does not vanish in the paraxial limit. Indeed, setting $c_{1} \rightarrow 1$, $c_{2} \rightarrow-1$, and $s_{i} \rightarrow 0$ in the above expressions, we obtain

$$
\mathcal{M}_{S}=2 g E_{1} E_{2} \delta_{\lambda_{1}, \lambda_{2}} e^{-i \lambda\left(\varphi_{1}-\varphi_{2}\right)},
$$

and, as the result, we get

$$
\begin{aligned}
\mathcal{J}_{S} & \propto \cos \left[\left(m_{1}-\lambda\right) \delta_{1}+\left(m_{2}-\lambda\right) \delta_{2}\right] \\
& =\cos \left(\ell_{1} \delta_{1}+\ell_{2} \delta_{2}\right),
\end{aligned}
$$

which coincides with the expression for twisted scalar annihilation [36]. Here, $\ell_{i}$ are the $z$ projections of the OAM of the two photons, which are approximately conserved in this limit. The sizable spin asymmetry $\sigma_{a}$ originates from the fact that, in the paraxial approximation, 


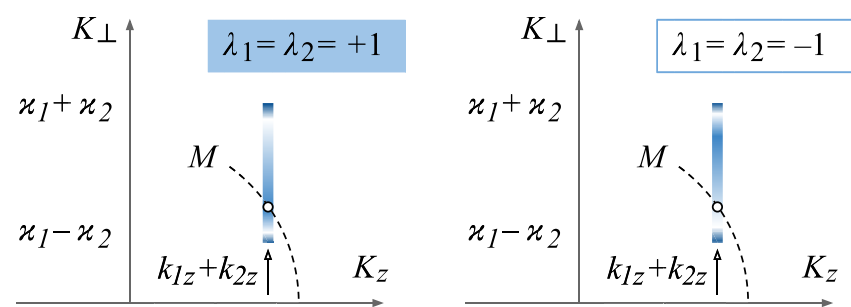

FIG. 2. The absolute value of the transverse momentum $K$ and the longitudinal momentum $K_{z}$ of the produced scalar resonance of mass $M$ in Bessel photon collision is uniquely reconstructed from the energy-momentum conservation for either helicity arrangement: $\lambda_{1}=\lambda_{2}=+1$ (left plot) and $\lambda_{1}=\lambda_{2}=-1$ (right plot). However, the magnitude of the cross section (shown as shades of blue) can be different due to different interference patterns, leading to an energy-dependent polarization asymmetry.

$\lambda=+1$ involves the OAM state $\ell=m-1$, while $\lambda=-1$ involves the OAM state $\ell^{\prime}=m+1=\ell+2$. The two states have different spatial distributions. As schematically illustrated by Fig. 2, the resonance production amplitude in collision of Bessel twisted photons involves interference between two plane wave amplitudes. Since this interference depends on the OAM values, one observes nonidentical cross sections $\sigma_{\lambda=+1}$ and $\sigma_{\lambda=-1}$.

For Bessel photon collisions, if the initial kinematics is fixed, then the longitudinal momentum of the produced particle $K_{z}$ and its modulus of the transverse momentum $K$ are also fixed. This uniquely defines $\delta_{1}$ and $\delta_{2}$ and, therefore, the exact position with respect to the interference fringes. However, if one performs the total energy scan, then $K$ and/or $K_{z}$ will vary, and one can slide across interference fringes and observe rapidly changing asymmetry $A$. In particular, one can choose a particular position on the fringe to enhance the asymmetry contrast $A$ as much as possible, that is, to achieve $\sigma_{\lambda=+1} \gg \sigma_{\lambda=-1}$ or vice versa. On the other hand, just as in the scalar case [36], one can anticipate that the Gaussian smearing of the pure Bessel states will reduce fringe visibility and the asymmetry contrast.

Figure 3 demonstrates the typical values of the asymmetry one can obtain for realistic twisted photon beams. For illustration, we take a narrow resonance with mass $M=0.8 \mathrm{GeV}$ and show the distribution of the cross sections $\sigma_{\lambda}$ and the asymmetry contrast $A$ over the total energy $E_{K}$ for the Gaussian-smeared twisted states with kinematic parameters

$$
\begin{aligned}
\left(m_{1}, m_{2}\right) & =(2,1), & & \bar{\varkappa}_{1}=0.1 \mathrm{GeV}, \\
\bar{\varkappa}_{2} & =0.2 \mathrm{GeV}, & & \bar{K}_{z}=-0.25 \mathrm{GeV},
\end{aligned}
$$

and with $\sigma_{i}=\bar{\varkappa}_{i} / 10$. Here and below, we use the notation $\bar{K}_{z} \equiv \bar{k}_{1 z}+\bar{k}_{2 z}$ with $\bar{k}_{i z}=\sqrt{E_{i}^{2}-\bar{\varkappa}_{i}^{2}}$. As we see, the asymmetry contrast remains very high, and as we scan over the total energy, it swings from almost -1 to +1 . This is an unprecedented sensitivity to the photon polarization in a process, which is fundamentally $P$ invariant.

\section{Helicity dependence: Fixed-C case}

Let us now adapt a different definition of what unpolarized twisted photons mean; see Sec. II D. When comparing $\gamma \gamma$ collisions with $\lambda_{1}=\lambda_{2}= \pm 1$, we now assume that $m_{1}$ and $m_{2}$ change accordingly, so that $\ell_{i} \equiv m_{i}-\lambda_{i}$ are fixed. In this case, in the paraxial approximation, $\mathcal{J}_{S}$ becomes independent of $\lambda$; see Eq. (41). However, beyond the paraxial approximation, the difference persists. Since the quantities $\mathcal{J}_{1}$ and $\mathcal{J}_{2}$ defined in Eq. (37) now depend on $\lambda$, we replace (36) with

$$
\mathcal{J}_{S}=e^{i\left(\ell_{1}-\ell_{2}\right) \varphi_{K}} \delta_{\lambda_{1}, \lambda_{2}} \frac{2 g E_{1} E_{2} \varkappa_{1} \varkappa_{2}}{\Delta} \cdot\left(\mathcal{J}_{1}^{\prime}+\lambda \mathcal{J}_{2}^{\prime}\right),
$$

where

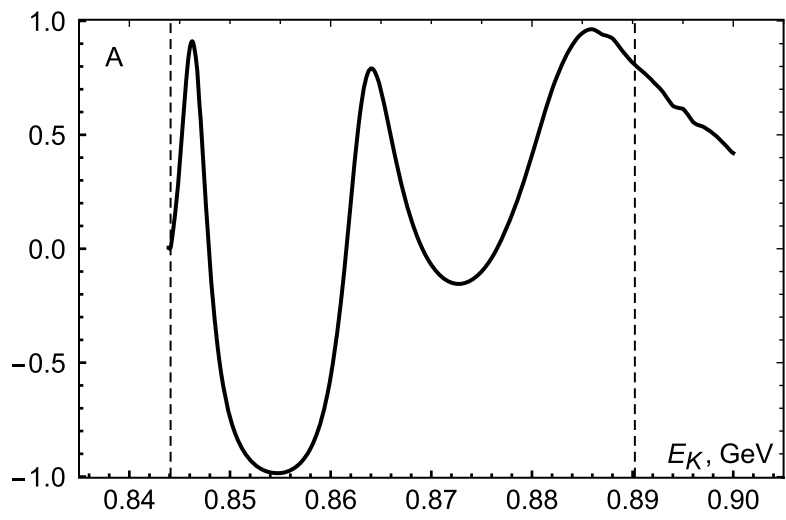

FIG. 3. Left: Cross section of the process $\gamma \gamma \rightarrow S$ with twisted photons with parameters (42) for two helicity configurations: $\lambda_{1}=$ $\lambda_{2}=+1$ (blue) and -1 (red). Right: the asymmetry contrast $A$ defined in Eq. (39). 


$$
\begin{aligned}
\mathcal{J}_{1}^{\prime}= & {\left[\frac{\left(1+c_{1}\right)\left(1-c_{2}\right)}{2}+\frac{\left(1-c_{1}\right)\left(1+c_{2}\right)}{2} \cos \left[2\left(\delta_{1}+\delta_{2}\right)\right]\right.} \\
& \left.-s_{1} s_{2} \cos \left(\delta_{1}+\delta_{2}\right)\right] \cos \left(\ell_{1} \delta_{1}+\ell_{2} \delta_{2}\right), \\
\mathcal{J}_{2}^{\prime}= & {\left[s_{1} s_{2}-\left(1-c_{1}\right)\left(1+c_{2}\right) \cos \left(\delta_{1}+\delta_{2}\right)\right] } \\
& \times \sin \left(\ell_{1} \delta_{1}+\ell_{2} \delta_{2}\right) \sin \left(\delta_{1}+\delta_{2}\right) .
\end{aligned}
$$

In the paraxial approximation $\theta_{1} \ll 1, \bar{\theta}_{2} \equiv \pi-\theta_{2} \ll 1$,

$$
\begin{aligned}
\mathcal{J}_{1}^{\prime} & \rightarrow 2 \cos \left(\ell_{1} \delta_{1}+\ell_{2} \delta_{2}\right), \\
\mathcal{J}_{2}^{\prime} & \rightarrow \theta_{1} \bar{\theta}_{2} \sin \left(\ell_{1} \delta_{1}+\ell_{2} \delta_{2}\right) \sin \left(\delta_{1}+\delta_{2}\right) \ll \mathcal{J}_{1}^{\prime},
\end{aligned}
$$

so that

$$
A \approx \theta_{1} \bar{\theta}_{2} \tan \left(\ell_{1} \delta_{1}+\ell_{2} \delta_{2}\right) \sin \left(\delta_{1}+\delta_{2}\right) \ll 1 .
$$

Thus, the nonzero asymmetry is suppressed by the small angles $\theta_{1}$ and $\bar{\theta}_{2}$, but it may be additionally enhanced if a suitable position on the fringe is selected.

The above two evaluations of the polarization asymmetry of the twisted photon collision cross section (39) and (46) differ significantly. The real experimental situation will probably lie in between. Indeed, even if one produces twisted photons using holographic plates, then one obtains, just behind the plate, a light field whose spatial distribution is not sensitive to its polarization. However, the light field evolves downstream and will certainly be different in the focal plane due to the intrinsic spin-orbital interaction of light [35], as we discussed in Sec. II D. Thus, the exact value of polarization asymmetry cannot be predicted without details of the experimental scheme.

However, the mere fact of spontaneous generation of a (sizable) polarization asymmetry in twisted photon collisions is beyond any doubt. This asymmetry is certainly absent in the usual plane photon-photon collision and represents a novel experimental tool offered by twisted photons.

\section{Detecting scalar-pseudoscalar mixing in unpolarized twisted $\gamma \gamma$ collisions}

In the previous subsections, we demonstrated that unpolarized twisted photon collision has a new intrinsic, adjustable degree of freedom, which is absent in the plane wave case: a difference between $\sigma_{\lambda=+1}$ and $\sigma_{\lambda=-1}$. We will now show how it can be applied to detect scalar-pseudoscalar mixing in a spin-0 resonance produced in collision of unpolarized twisted photons. This is our first example of an observable which up to now was considered accessible only in production of polarized photons or via the subsequent decays of the resonance produced.

Let us begin by considering production of a pseudoscalar particle $P$ in collision of two twisted photons. The coupling is generated by the effective Lagrangian

$$
\mathcal{L}_{P}=i \frac{g}{4} F^{\mu \nu} \tilde{F}_{\mu \nu} P
$$

where $\tilde{F}_{\mu \nu}=\epsilon_{\mu \nu \rho \sigma} F^{\rho \sigma} / 2$ is the dual electromagnetic field strength tensor. It generates the following plane wave helicity amplitude

$$
\mathcal{M}_{P}=i g \epsilon_{\mu \nu \rho \sigma} k_{1}^{\mu} k_{2}^{\nu} e_{1}^{\rho} e_{2}^{\sigma} .
$$

Working in the same Coulomb gauge, one can evaluate this amplitude explicitly to find the same structure as for the true scalar (32) times the overall helicity factor $\lambda$,

$$
\mathcal{M}_{P}=\lambda \mathcal{M}_{S}
$$

where for simplicity we used the same coupling constant $g$ in both cases. For twisted photons, one concludes that $\mathcal{J}_{P}=\lambda \mathcal{J}_{S}$, which generates exactly the same cross section as in the scalar case. Thus, in the total production cross section, the pure scalar and pure pseudoscalar cases are as indistinguishable for twisted photon collisions as for plane waves.

Next, suppose the spin- 0 particle produced does not possess definite parity. Then, its production amplitude is

$$
\mathcal{M}=a \mathcal{M}_{S}+b \mathcal{M}_{P}=(a+\lambda b) \mathcal{M}_{S}
$$

The (complex) coefficients $a$ and $b$ describe the scalarpseudoscalar coupling of the particle to two photons. In the usual plane wave collision with circularly polarized photons, the cross section is

$$
\sigma_{\lambda} \propto\left[|a|^{2}+|b|^{2}+2 \lambda \operatorname{Re}\left(a^{*} b\right)\right] \cdot\left|\mathcal{M}_{S}\right|^{2} .
$$

By measuring the unpolarized production cross section, one can only reveal the overall production intensity $|a|^{2}+|b|^{2}$ but not detect the amount of scalar-pseudoscalar mixing. It can be detected, in the plane wave collisions, only if one performs experiments with polarized photons and measures various spin asymmetries. For example, circularly polarized photons give access to $\sigma_{+}-\sigma_{-} \propto \operatorname{Re}\left(a^{*} b\right)$; additional information can be recovered with linearly polarized photons. This is a standard way to probe the parity properties of the produced resonance.

Remarkably, twisted photons offer access to this scalarpseudoscalar mixing even with unpolarized twisted photons. Using the fixed- $m$ convention for unpolarized twisted light, we obtain the twisted production amplitude as

$$
\begin{aligned}
\mathcal{J} & =\left(\mathcal{J}_{1}+\lambda \mathcal{J}_{2}\right)(a+\lambda b) \\
& =\left(a \mathcal{J}_{1}+b \mathcal{J}_{2}\right)+\lambda\left(b \mathcal{J}_{1}+a \mathcal{J}_{2}\right),
\end{aligned}
$$

with helicity-independent $\mathcal{J}_{1}, \mathcal{J}_{2}$ given in (37). Squaring it and averaging over the initial photon helicities, we obtain the unpolarized cross section as 
$\sigma_{+}+\sigma_{-} \propto\left(\mathcal{J}_{1}^{2}+\mathcal{J}_{2}^{2}\right)\left(|a|^{2}+|b|^{2}\right)+4 \mathcal{J}_{1} \mathcal{J}_{2} \operatorname{Re}\left(a^{*} b\right)$.

Thus, even for unpolarized twisted photons, the cross section contains a term which is sensitive to the magnitude of scalar-pseudoscalar mixing.

This contributions can be extracted by a scan of the total cross section over the collision energy. Indeed, $\mathcal{J}_{1}$ and $\mathcal{J}_{2}$ in (37) have different dependence on $\delta_{1}$ and $\delta_{2}$. Therefore, the $\mathcal{J}_{1} \mathcal{J}_{2}$ term exhibits a different interference pattern than $\mathcal{J}_{1}^{2}+\mathcal{J}_{2}^{2}$ as one scans over the allowed energy interval. To illustrate this effect, we present in Fig. 4 the total energy scan of the cross section for a pure scalar or pseudoscalar (which are indistinguishable) and for the case of their maximal mixing with $a=b=1 / \sqrt{2}$. One sees a clear difference of the interference fringes in these two cases. This particular plot corresponds to the kinematic parameters (42), but there certainly exists ample room for improving the discriminating power of this measurement.

\section{Spin physics with unpolarized twisted photons: $f_{2}$ example}

Let us now see what unpolarized twisted photons can do for spin-2 resonances such as the $f_{2}(1270)$ meson. In the usual plane wave case, unpolarized $\gamma \gamma$ collisions produce an equal amount of $\lambda_{K}$ and $-\lambda_{K}$ polarization states. But in unpolarized twisted photon collisions, one can selectively produce different helicity states by adjusting the total collision energy in order to stay at an appropriate interference fringe.

To see how it works, we begin by reviewing basic features of the $\gamma \gamma \rightarrow f_{2}$ process. The tensor meson $f_{2}$ with helicity $\lambda_{K}$ is described with the symmetric polarization tensor $T_{\mu \nu}^{\left(\lambda_{K}\right)}$ orthogonal to its 4-momentum: $T_{\mu \nu}^{\left(\lambda_{K}\right)} K^{\nu}=0$.

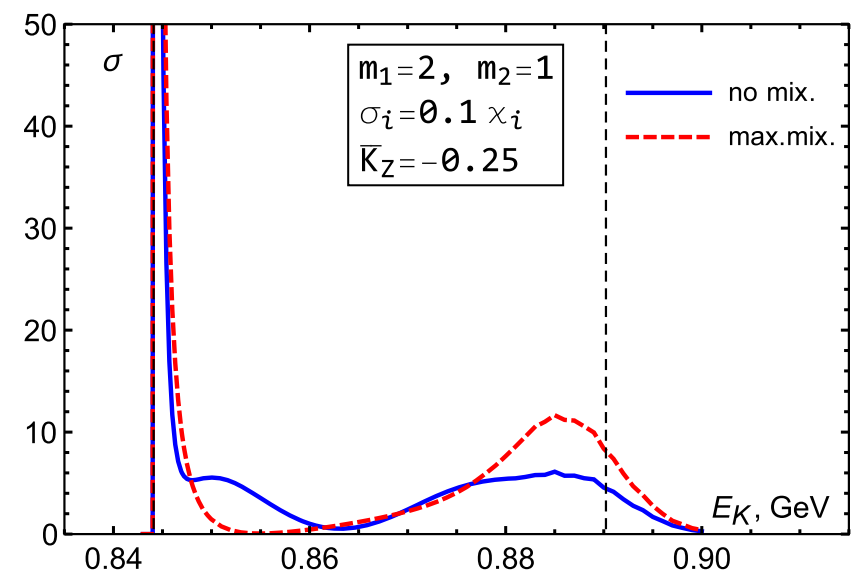

FIG. 4. The total production cross section of a spin-0 state in twisted $\gamma \gamma$ collision with parameters (42) with Gaussian-smeared twisted photons $\sigma_{i}=\varkappa_{i} / 10$. Shown are cases without mixing (blue solid line) and with maximal scalar-pseudoscalar mixing (red dashed line).
It has five polarization states, with $\lambda_{K}$ spanning from -2 to +2 . They are constructed with the three polarization vectors $e_{\mu}^{(\lambda)}, \lambda= \pm 1,0$, orthogonal to $K^{\mu}$ : the vectors $e_{\mu}^{( \pm 1)}=\left(0, \mathbf{e}_{\mathbf{k} \lambda}\right)$ can be taken as defined in Eq. (12), while $e_{\mu}^{(0)}=\gamma_{K}\left(\beta_{K}, \mathbf{n}_{K}\right)$, where $\beta_{K}$ and $\gamma_{K}$ are the standard kinematic parameters for the produced meson and $\mathbf{n}_{K}$ is the unit vector in the direction $\mathbf{K}$. The explicit expressions for the five polarization states of the spin-2 meson are

$$
\begin{aligned}
T_{\mu \nu}^{( \pm 2)} & =e_{\mu}^{( \pm)} e_{\nu}^{( \pm)}, \quad T_{\mu \nu}^{( \pm 1)}=\frac{1}{\sqrt{2}}\left(e_{\mu}^{( \pm)} e_{\nu}^{(0)}+e_{\mu}^{(0)} e_{\nu}^{( \pm)}\right), \\
T_{\mu \nu}^{(0)} & =\frac{1}{\sqrt{6}}\left(e_{\mu}^{(+)} e_{\nu}^{(-)}+e_{\mu}^{(-)} e_{\nu}^{(+)}+2 e_{\mu}^{(0)} e_{\nu}^{(0)}\right) .
\end{aligned}
$$

The interaction between the two photons and the $f_{2}$ meson is generated by the Lagrangian $g F_{\rho \mu} F_{\rho \nu} T_{\mu \nu} / 2$, which gives rise to the following plane wave $\gamma \gamma \rightarrow f_{2}$ amplitude:

$$
\begin{aligned}
\mathcal{M}= & g\left[\left(k_{1} k_{2}\right) e_{1 \mu} e_{2 \nu}+\left(e_{1} e_{2}\right) k_{1 \mu} k_{2 \nu}-\left(k_{1} e_{2}\right) e_{1 \mu} k_{2 \nu}\right. \\
& \left.-\left(k_{2} e_{1}\right) k_{1 \mu} e_{2 \nu}\right]\left(T_{\mu \nu}^{\left(\lambda_{K}\right)}\right)^{*} .
\end{aligned}
$$

Once again, all scalar products are understood as products of 4-vectors. The polarization vectors for the two photons $e_{1}$ and $e_{2}$ depend on their helicities $\lambda_{1}$ and $\lambda_{2}$ and are orthogonal to their respective momenta $k_{1}$ and $k_{2}$. We work in the Coulomb gauge and use the same vectors (12). As in the case of spin- 0 production, we need the amplitude in the generic kinematics. This is why we do not assume that $\left(k_{1} e_{2}\right)=\left(e_{1} k_{2}\right)=0$ and keep all four terms in (55).

In the plane wave case, one can switch to the center of motion reference frame, in which the produced $f_{2}$ is at rest and the photons are along the $z$ axis. All polarization vectors in this case can be identified with the vectors $\chi_{\lambda_{i}}$ defined in (9). If one chooses the same axis $z$ to define the helicity of the $f_{2}$ meson, then the helicity amplitudes will take the following very simple form:

$$
\mathcal{M}_{\lambda_{K}= \pm 2}=2 g E^{2} \delta_{\lambda_{1}, \pm} \delta_{\lambda_{2}, \mp} \cdot
$$

That is, only \pm 2 polarization states can be produced and only for opposite photon helicities. If the two photons have different energies $E_{1} \neq E_{2}$ but their momenta are still along axis $z$, the final meson moves along the same axis and has helicity \pm 2 depending on the initial photon helicities. For unpolarized photon beams, the final $f_{2}$ meson is, of course, also unpolarized.

For unpolarized twisted photons, the produced $f_{2}$ meson has a nonvanishing average helicity. To illustrate the main idea, we stick to the paraxial approximation for the two photons, $\theta_{1} \rightarrow 0$ and $\theta_{2} \rightarrow \pi$, while keeping the polar angle $\theta_{K}$ of the produced $f_{2}$ meson generic (28). In the paraxial limit, the helicity amplitudes with $\lambda_{K}= \pm 2$ dominate, and their expressions take a simple form. For $\lambda_{K}=+2$, we get 


$$
\begin{aligned}
& \left(\lambda_{1}, \lambda_{2}\right)=(+,-): \mathcal{M}=\frac{g}{2} E_{1} E_{2}\left(1+\cos \theta_{K}\right)^{2} e^{-i\left(\varphi_{1}+\varphi_{2}-2 \varphi_{K}\right)}, \\
& \left(\lambda_{1}, \lambda_{2}\right)=(-,+): \mathcal{M}=\frac{g}{2} E_{1} E_{2}\left(1-\cos \theta_{K}\right)^{2} e^{i\left(\varphi_{1}+\varphi_{2}-2 \varphi_{K}\right)},
\end{aligned}
$$

which gives $\mathcal{J}$, up to a common prefactor, of the form

$$
\begin{aligned}
\left(\lambda_{1}, \lambda_{2}\right)=(+,-): \mathcal{J} \propto & \left(1+\cos \theta_{K}\right)^{2} \\
& \times \cos \left(m_{1} \delta_{1}+m_{2} \delta_{2}-\delta_{1}+\delta_{2}\right), \\
\left(\lambda_{1}, \lambda_{2}\right)=(-,+): \mathcal{J} \propto & \left(1-\cos \theta_{K}\right)^{2} \\
& \times \cos \left(m_{1} \delta_{1}+m_{2} \delta_{2}+\delta_{1}-\delta_{2}\right) .
\end{aligned}
$$

For $\lambda_{K}=-2$, we get

$$
\begin{aligned}
\left(\lambda_{1}, \lambda_{2}\right)=(+,-): \mathcal{J} \propto & \left(1-\cos \theta_{K}\right)^{2} \\
& \times \cos \left(m_{1} \delta_{1}+m_{2} \delta_{2}-\delta_{1}+\delta_{2}\right), \\
\left(\lambda_{1}, \lambda_{2}\right)=(-,+): \mathcal{J} \propto & \left(1+\cos \theta_{K}\right)^{2} \\
& \times \cos \left(m_{1} \delta_{1}+m_{2} \delta_{2}+\delta_{1}-\delta_{2}\right) .
\end{aligned}
$$

It is immediately seen that for $\cos \theta_{K}=0$ the two polarization states $\lambda_{K}= \pm 2$ are produced in equal amounts by the opposite photon helicities. However, at $\cos \theta_{K} \neq 0$, this equivalence breaks down. Calculating $|\mathcal{J}|^{2}$ and averaging it over the initial photon helicities, we get the following unpolarized cross section for $\lambda_{K}= \pm 2$ :

$$
\begin{aligned}
\sigma_{\lambda_{K}= \pm 2} \propto & \left(1+6 \cos ^{2} \theta_{K}+\cos ^{4} \theta_{K}\right) \\
& \times\left[1+\cos \left(2 m_{1} \delta_{1}+2 m_{2} \delta_{2}\right) \cos \left(2 \delta_{1}-2 \delta_{2}\right)\right] \\
& +2 \lambda_{K} \cos \theta_{K}\left(1+\cos ^{2} \theta_{K}\right) \cdot \sin \left(2 m_{1} \delta_{1}+2 m_{2} \delta_{2}\right) \\
& \times \sin \left(2 \delta_{1}-2 \delta_{2}\right)
\end{aligned}
$$

Thus, even if the twisted photons are unpolarized, we do see a difference between $\left|\mathcal{J}_{+2}\right|^{2}$ and $\left|\mathcal{J}_{-2}\right|^{2}$ and, therefore, between the production cross sections:

$$
\begin{aligned}
\sigma_{\lambda_{K}=+2}-\sigma_{\lambda_{K}=-2} & \propto \cos \theta_{K}\left(1+\cos ^{2} \theta_{K}\right) \cdot \sin \left(2 m_{1} \delta_{1}+2 m_{2} \delta_{2}\right) \\
& \times \sin \left(2 \delta_{1}-2 \delta_{2}\right) .
\end{aligned}
$$

For the longitudinally balanced collision, $k_{1 z}+k_{2 z}=0$, the emission angle is $\theta_{K}=\pi / 2$, and this spin asymmetry vanishes. But for an off-balanced situation with a generic $\theta_{K}$, the asymmetry is present and, in general, not small. Scanning the total energy of the collision and adjusting $m_{i}$, one can find optimal conditions when one produces preferentially +2 polarized states over -2 states.

We skip the numerical study of this effect for the $\gamma \gamma \rightarrow f_{2}$ production because in the following section we will study it at length for vector resonances produced in the unpolarized twisted $e^{+} e^{-}$annihilation.

\section{SPIN ASYMMETRY IN TWISTED $e^{+} e^{-}$ANNIHILATION}

Access to spin-dependent observables in unpolarized inclusive cross section can also be expected from $e^{+} e^{-}$ annihilation, provided both the electron and the positron are twisted. As discussed in Sec. II D, an unpolarized twisted electron beam can be defined as an equal flux of twisted electrons with helicities $\zeta=+1 / 2$ and $\zeta=-1 / 2$ and either with fixed total angular momentum $m$ or fixed $\ell=m-\zeta$. One can then calculate production of a vector meson with helicity $\lambda_{K}= \pm 1$ with unpolarized twisted electrons and observe a nonzero asymmetry $\sigma_{\lambda_{K}=+1}-\sigma_{\lambda_{K}=-1}$. In this section, we will adopt the former definition of the unpolarized twisted electrons (fixed $m$ ), where a large effect is expected. What is actually feasible in experiment will eventually depend on the exact scheme of preparation of twisted electrons and positrons.

Helicity amplitudes for vector (spin-1) meson production in the plane wave annihilation process $e^{-}\left(k_{1}, \zeta_{1}\right) e^{+}\left(k_{2}, \zeta_{2}\right) \rightarrow$ $V\left(K, \lambda_{K}\right)$ can defined as

$$
\mathcal{M}_{\zeta_{1} \zeta_{2} \lambda}=g \bar{v}_{\zeta_{2}}\left(k_{2}\right) \gamma_{\mu} u_{\zeta_{1}}\left(k_{1}\right) V_{\lambda_{K}}^{\mu *}(K)
$$

Here, for the sake of simplicity, we assumed that the vector meson couples to the same $\bar{v} \gamma^{\mu} u$ current as the photon. For realistic vector mesons, the current may differ according to whether the meson represents an $S$-wave or $D$-wave state of the quark-antiquark pair [40], but investigating this issue goes beyond the scope of the present paper.

The polarization vector $V_{\lambda_{K}}^{\mu}(k)$ is constructed in the same way as previously,

$$
V_{ \pm 1}^{\mu}=\left(0, \mathbf{e}_{\mathbf{k}, \pm 1}\right), \quad V_{0}^{\mu}=\gamma_{K}\left(\beta_{K}, \mathbf{n}_{K}\right),
$$

where $\mathbf{e}_{\mathbf{k} \lambda}$ is defined in Eq. (12). To simplify the calculations without losing the main features, we assume the electrons and positrons to be ultrarelativistic and neglect their mass. Then, one observes that the nonzero amplitudes exist only for $\zeta_{1}=-\zeta_{2} \equiv \zeta$,

$$
\mathcal{M}_{\zeta_{1} \zeta_{2} \lambda_{K}}=-2 g \delta_{\zeta_{1},-\zeta_{2}} \sqrt{E_{1} E_{2}} \cdot T_{\mu}^{(\zeta)} V_{\lambda_{K}}^{\mu *}
$$

where

$$
\begin{aligned}
T_{0}^{(\zeta)} & =w_{2}^{(\zeta) \dagger} w_{1}^{(\zeta)}=c_{1} c_{2} e^{i \zeta\left(\varphi_{2}-\varphi_{1}\right)}+s_{1} s_{2} e^{-i \zeta\left(\varphi_{2}-\varphi_{1}\right)}, \\
T_{3}^{(\zeta)} & =2 \zeta w_{2}^{(\zeta) \dagger} \sigma_{3} w_{1}^{(\zeta)}=c_{1} c_{2} e^{i \zeta\left(\varphi_{2}-\varphi_{1}\right)}-s_{1} s_{2} e^{-i \zeta\left(\varphi_{2}-\varphi_{1}\right)}, \\
T_{1}^{(\zeta)} & =2 \zeta w_{2}^{(\zeta) \dagger} \sigma_{1} w_{1}^{(\zeta)}=c_{2} s_{1} e^{i \zeta\left(\varphi_{2}+\varphi_{1}\right)}+s_{2} c_{1} e^{-i \zeta\left(\varphi_{2}+\varphi_{1}\right)}, \\
T_{2}^{(\zeta)} & =2 \zeta w_{2}^{(\zeta) \dagger} \sigma_{2} w_{1}^{(\zeta)} \\
& =-2 i \zeta\left[c_{2} s_{1} e^{i \zeta\left(\varphi_{2}+\varphi_{1}\right)}-s_{2} c_{1} e^{-i \zeta\left(\varphi_{2}+\varphi_{1}\right)}\right] .
\end{aligned}
$$

One can verify that $T_{\mu}^{(\zeta)} k_{1}^{\mu}=T_{\mu}^{(\zeta)} k_{2}^{\mu}=0$. We remind the reader that, for fermions, the shorthand notation is to be understood as $c_{i} \equiv \cos \left(\theta_{i} / 2\right)$ and $s_{i} \equiv \sin \left(\theta_{i} / 2\right)$. 
These helicity amplitudes can be evaluated for generic kinematics, but the main features again can be illustrated in the paraxial approximation, $\theta_{1} \rightarrow 0, \theta_{2} \rightarrow \pi$, so that only terms with $c_{1} \rightarrow 1$ and $s_{2} \rightarrow 1$ survive. The polar angle $\theta_{K}$ of the produced resonance is kept generic. The surviving helicity amplitudes correspond to production of $\lambda_{K}= \pm 1$ states:

$\mathcal{M}_{\zeta,-\zeta, \lambda_{K}}=-g \sqrt{2 E_{1} E_{2}} e^{-i \zeta\left(\varphi_{2}+\varphi_{1}-2 \varphi_{K}\right)} \cdot\left(\lambda_{K} \cos \theta_{K}+2 \zeta\right)$.

When passing from plane waves to twisted Bessel states, we use the same expression for $\mathcal{J}$ as in Eq. (26), with halfinteger $m_{1}, m_{2}$. The plane wave amplitudes $\mathcal{M}_{a}$ and $\mathcal{M}_{b}$ for the two kinematic configurations are given by Eq. (66), in which the azimuthal angles take their values set by Eqs. (24). The resulting expression for the twisted amplitude is

$$
\begin{aligned}
\mathcal{J}_{\zeta,-\zeta, \lambda_{K}}= & -g \sqrt{2 E_{1} E_{2}} e^{i\left(m_{1}-m_{2}\right) \varphi_{K}} \frac{\varkappa_{1} \varkappa_{2}}{\Delta} \cdot\left(\lambda_{K} \cos \theta_{K}+2 \zeta\right) \\
& \times \cos \left[m_{1} \delta_{1}+m_{2} \delta_{2}-\zeta\left(\delta_{1}-\delta_{2}\right)\right] .
\end{aligned}
$$

Using the fixed- $m$ definition of the unpolarized electron and positron beams, we obtain the following unpolarized twisted $e^{+} e^{-}$cross section in the paraxial approximation:

$\sigma_{\lambda_{K}= \pm 1} \propto\left(1+\cos ^{2} \theta_{K}\right)\left[1+\cos \left(2 m_{1} \delta_{1}+2 m_{2} \delta_{2}\right) \cos \left(\delta_{1}-\delta_{2}\right)\right]$

$$
+2 \lambda_{K} \cos \theta_{K} \sin \left(2 m_{1} \delta_{1}+2 m_{2} \delta_{2}\right) \sin \left(\delta_{1}-\delta_{2}\right) .
$$

This result is similar to the $f_{2}$-meson production in unpolarized twisted photon collision: for a generic $\theta_{K} \neq \pi / 2$, there is a clear imbalance between $\lambda_{K}=+1$ and $\lambda_{K}=-1$ production cross sections, which depends on the exact position with respect to the interference fringes. The asymmetry contrast can be defined as

$$
A=\frac{\sigma_{\lambda_{K}=+1}-\sigma_{\lambda_{K}=-1}}{\sigma_{\lambda_{K}=+1}+\sigma_{\lambda_{K}=-1}+\sigma_{\lambda_{K}=0}},
$$

and in the paraxial limit, it can be approximated as

$A \approx \frac{2 \cos \theta_{K}}{1+\cos ^{2} \theta_{K}} \cdot \frac{\sin \left(2 m_{1} \delta_{1}+2 m_{2} \delta_{2}\right) \sin \left(\delta_{1}-\delta_{2}\right)}{1+\cos \left(2 m_{1} \delta_{1}+2 m_{2} \delta_{2}\right) \cos \left(\delta_{1}-\delta_{2}\right)}$.

Thus, for the production angles $\theta_{K}$ far away from $\pi / 2$, the asymmetry can be rather sizable. Notice that in the paraxial limit $\sigma_{\lambda_{K}=0} \rightarrow 0$.
These paraxial estimates can be corroborated with numerical calculations based on the exact expression for the helicity amplitudes (65). To give a concrete example, we consider production of the $J / \psi$ meson with mass $M=$ $3.1 \mathrm{GeV}$ in the unpolarized twisted $e^{+} e^{-}$annihilation with the following parameters:

$$
\begin{array}{rlrl}
\varkappa_{1} & =0.2 \mathrm{GeV}, & \varkappa_{2}=0.1 \mathrm{GeV}, \\
m_{1}=5 / 2, & m_{2}=1 / 2 .
\end{array}
$$

To define the scan trajectory on the $\left(E_{1}, E_{2}\right)$ plane, we will first choose the electron energy $E_{1}$ and then scan over a range of the positron energies $E_{2}$ plotting the cross section as a function of the final particle polar angle $\theta_{K}$ calculated from Eq. (28).

In Fig. 5, we plot the polarization asymmetry for pure Bessel beams with different values of the electron energy $E_{1}$ ranging from 1.3 to $1.8 \mathrm{GeV}$ and with $E_{2}$ scanned in a certain range for each $E_{1}$. We see that the exact numerical results are very well approximated by the paraxial limit for the kinematic configuration away from $\theta_{K}=\pi / 2$. The sign of the predominant polarization asymmetry is clearly correlated with the forward or backward production hemisphere. For a fixed energy $E_{1}$, the curve is nonsymmetric, which implies that, even if electron and positron beams have certain energy spread, the overall polarization asymmetry will persist.

We stress that the distributions shown in Fig. 5 are not to be understood as the angular distribution in a single fixedenergy experiment. A single experiment with fixed energies $E_{1}$ and $E_{2}$ will correspond to one specific angle $\theta_{K}$ with its cross section and polarization. The plots in Fig. 5 represent the evolution of the polar production angle and the correlated evolution of the spin asymmetry value as one scans over the initial positron energy, keeping the electron

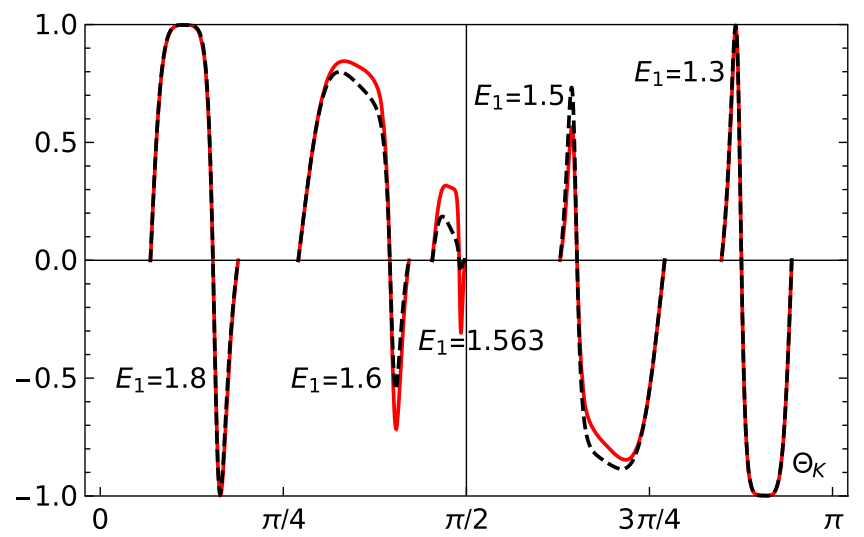

FIG. 5. The polarization asymmetry $A$ in Eq. (70) as a function of the final particle polar angle $\theta_{K}$ with the parameters given in (72) and for several choices of the electron energy $E_{1}$ given in $\mathrm{GeV}$. The black dashed lines are the asymmetries in the paraxial limit, while the red solid curves represent the exact result without including the $\sigma_{\lambda_{K}=0}$ contribution to Eq. (70). 
energy fixed. They tell us that selecting a point near the plateau would produce almost $100 \%$ polarized meson beam even with unpolarized initial electron and positron beams.

Since the exact Bessel beams are not normalizable, we model the realistic situation by smearing over the initial $\varkappa_{i}$ with the parameters

$\bar{\varkappa}_{1}=0.2 \mathrm{GeV}, \quad \bar{\varkappa}_{2}=0.1 \mathrm{GeV}, \quad \sigma_{i}=\bar{\varkappa}_{i} / 5$,

$E_{1}=1.8 \mathrm{GeV}, \quad E_{2}=1.338 \mathrm{GeV}$,

and for the angular momentum values $m_{1}=5 / 2$ and $m_{2}=1 / 2$. Although we now fix the energies of both incoming particles, smearing over $\varkappa_{i}$ produces a distribution over a range of angles $\theta_{K}$ in a single experiment. We also take into account the finite width of the produced resonance and evaluate the differential cross section weighted with the corresponding Breit-Wigner factor with the width $\Gamma=93 \mathrm{keV}$,

$$
\begin{aligned}
\frac{d \sigma}{d s d \cos \theta_{K}} & \left.\sim \sqrt{E_{K}^{2}-s \mid}\langle\mathcal{J}\rangle\right|^{2} \frac{1}{\pi} \frac{M \Gamma}{\left(s-M^{2}\right)^{2}+M^{2} \Gamma^{2}}, \\
\frac{d \sigma}{d \cos \theta_{K}} & \sim \frac{1}{\sqrt{E_{K}^{2}-M^{2}}} \int_{0}^{E_{K}} d s \frac{d \sigma}{d s d \cos \theta_{K}},
\end{aligned}
$$

where $s \equiv K_{\mu} K^{\mu}=E_{K}^{2}-K_{z}^{2}-K^{2}$ is the 4-momentum squared of the final particle. We stress that, unlike in the plane wave collision, the variable $s$ is not fixed by the initial state kinematics. Even when $E_{i}$ and $\varkappa_{i}$ are fixed, resonances with masses within a certain interval can be produced [36]. For resonances with finite width, this intrinsic massspectrometric feature of the twisted particle collision manifests itself as an $s$ distribution of the cross section. The dependence of the matrix element on the final particle parameters comes through $s$ and the polar angle $\theta_{K}$.

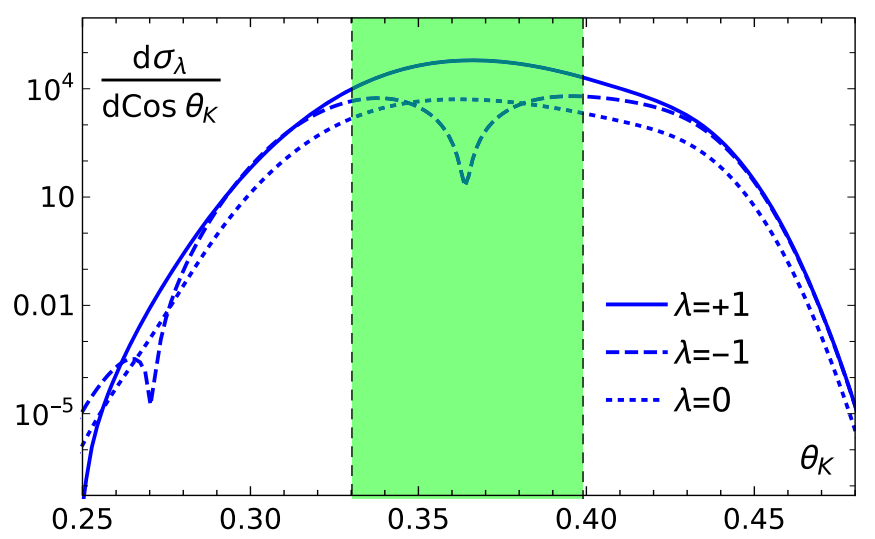

In Fig. 6, we show the resulting differential cross sections $d \sigma_{\lambda_{K}} / d \cos \theta_{K}$ for all three polarization states $\lambda_{K}= \pm 1,0$. The left plot shows these cross sections with zero width in the log scale, while the right plot, presenting the same functions in the linear scale, illustrates the minor effect of the nonzero width. The green band indicates the angular range which saturates the cross section.

As one sees, the cross section around the peak is strongly dominated by the polarization state $\lambda_{K}=+1$, with $\mathrm{a} \approx 10 \%$ admixture of the $\lambda_{K}=0$ state and even smaller contribution from $\lambda_{K}=-1$. This is not a coincidence but is a result of our choice of the kinematic parameters (73). Certainly, by adjusting these parameters, one can arrange for a situation with $\lambda_{K}=-1$ production strongly dominating over $\lambda_{K}=+1$. Thus, we obtain a remarkable result: we can produce almost fully polarized vector mesons in unpolarized twisted $e^{+} e^{-}$annihilation.

The polarization purity of the produced resonance can be quantified by the differential asymmetry $A\left(\theta_{K}\right)$ defined as

$$
A\left(\theta_{K}\right)=\left(\frac{d \sigma_{\lambda_{K}=+1}}{d \cos \theta_{K}}-\frac{d \sigma_{\lambda_{K}=-1}}{d \cos \theta_{K}}\right) / \sum_{\lambda_{K}} \frac{d \sigma_{\lambda_{K}}}{d \cos \theta_{K}} .
$$

This quantity is plotted in Fig. 7. Although the smearing effects reshape the angular profile of the asymmetry, it still rises as high as $90 \%$.

In the above numerical example, we used a very narrow resonance. Let us now see how the picture changes if one considers a wide resonance such as $\rho$ meson with mass $M_{\rho}=0.775 \mathrm{GeV}$ and width $\Gamma_{\rho}=0.149 \mathrm{GeV}$. We take the angular momentum values $m_{1}=5 / 2$ and $m_{2}=1 / 2$ and the following kinematical parameters:

$$
\begin{array}{ll}
\bar{\varkappa}_{1}=0.2 \mathrm{GeV}, & \bar{\varkappa}_{2}=0.1 \mathrm{GeV}, \quad \sigma_{i}=\bar{\varkappa}_{i} / 5, \\
E_{1}=0.6 \mathrm{GeV}, & E_{2}=0.258 \mathrm{GeV} .
\end{array}
$$

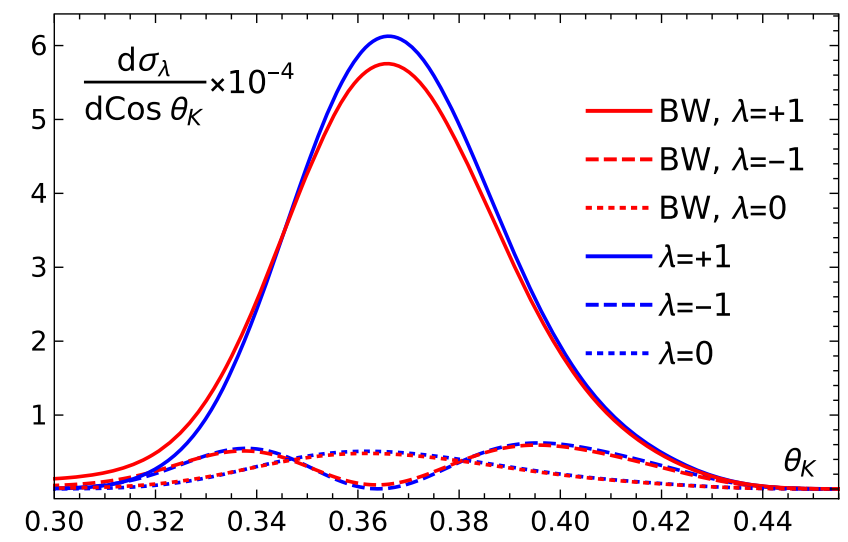

FIG. 6. Left: Distribution over the polar angles $\theta_{K}$ of the production of the $J / \psi$ meson in the zero-width approximation in the unpolarized twisted $e^{+} e^{-}$annihilation with kinematic parameters as in Eq. (73). Solid, dashed, and short-dashed lines show the cross sections for $\lambda_{K}=+1,-1$, and 0 , respectively. Right: the same cross sections in linear scale. The red curves include finite width effects according to Eq. (75), while the blue curves correspond to the zero width. The green band denotes the angular interval, which gives the main contribution to the cross section. 


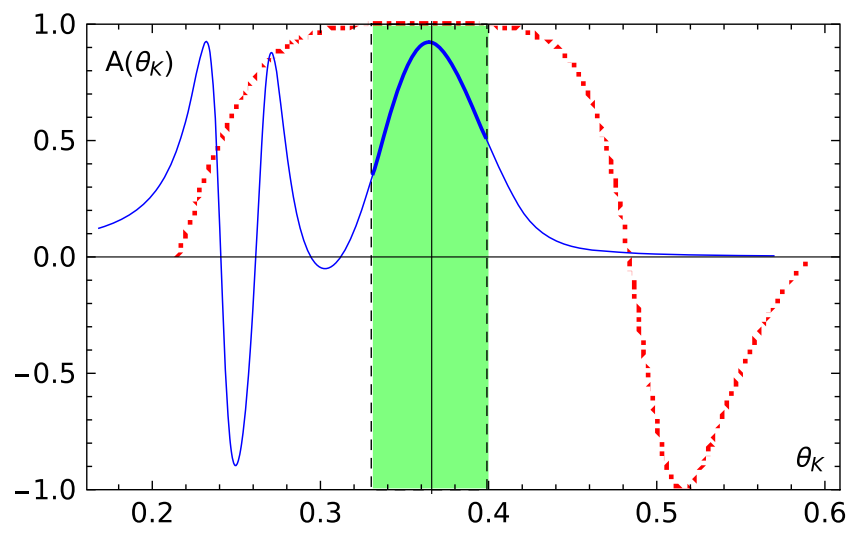

FIG. 7. The differential polarization asymmetry $A$, Eq. (76), as a function of the polar angle $\theta_{K}$ of the produced resonance. The red dotted line represent the result for the pure Bessel beams and is identical to the leftmost curve in Fig. 5. The blue solid line represents the asymmetry with smearing effects taken into account and with fixed energies of initial particles. The green band is the same as in Fig. 6, left. The vertical line around $\theta_{K} \approx 0.36$ represents the angle with $K_{z}=\bar{k}_{1 z}+\bar{k}_{2 z}$, where $\bar{k}_{i z}=\sqrt{E_{i}^{2}-\bar{\varkappa}_{i}^{2}}$.

In Fig. 8, we show the angular distribution of the $\rho$ production cross section in unpolarized twisted $e^{+} e^{-}$ annihilation for the three helicities of the produced meson. The dramatic broadening effect of the large width is evident. Nevertheless, the cross sections with $\lambda_{K}=+1$ and -1 differ significantly, so that the value of the asymmetry is nonzero and rather large.

The effect becomes even more pronounced if one studies the same angular distribution for selected values of $\sqrt{s}$. We remind the reader that, even when initial kinematical parameters $E_{i}$ and $\varkappa_{i}$ are fixed, the invariant mass of the produced resonance is not fixed and can vary within certain range [36]. However, since $\rho$ decays to the $\pi \pi$ system, the detector can reconstruct the invariant mass and the direction

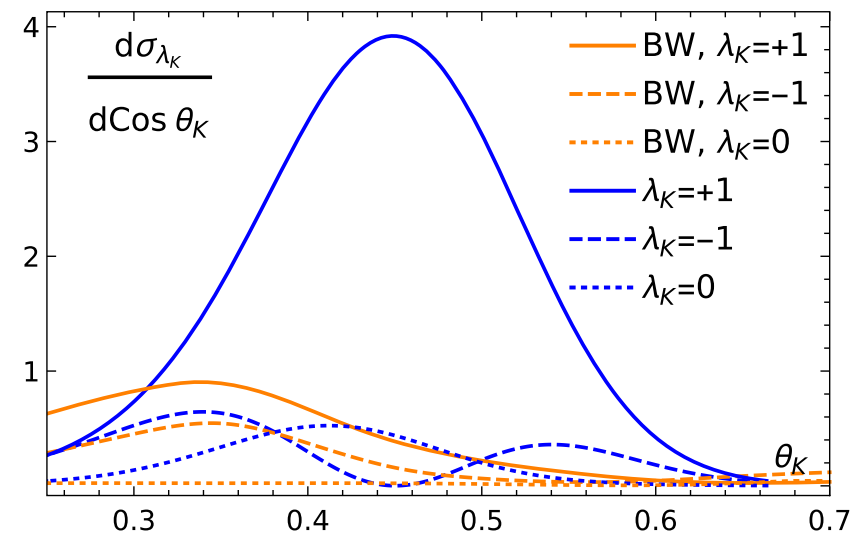

FIG. 8. The cross sections (in arbitrary units) as in Fig. 6, right, but for the wide $\rho$ meson. The lighter orange curves include finite width effects according to Eq. (75), while the blue curves correspond to the zero width. of the produced $\rho$ meson, enabling us to plot the fixed $\sqrt{s}=M_{\pi \pi}$ slice of the angular distribution.

The resulting differential cross sections $d \sigma_{\lambda_{K}} /\left(d s d \cos \theta_{K}\right)$ are presented in Fig. 9 together with differential polarization asymmetry for two values of invariant mass $\sqrt{s}=$ $0.777 \mathrm{GeV}$ and $\sqrt{s}=0.755 \mathrm{GeV}$. The left plot shows these cross sections for all three polarization states $\lambda_{K}= \pm 1,0$, while the right plot demonstrates the polarization asymmetry. The curves clearly show that, at fixed initial energy of the $e^{+} e^{-}$collision, the polarization of the produced meson dramatically depends on the production angle. For example, at $\sqrt{s}=0.777 \mathrm{GeV}$ (light red curves), the asymmetry $A$ reaches the values of $90 \%$ around $\theta_{K}=0.42$. By slightly lowering the collision energy to $\sqrt{s}=0.755 \mathrm{GeV}$ (dark blue curves), one completely reverses the situation: now the polarization state $\lambda_{K}=-1$ dominates, and the asymmetry reaches $-85 \%$ in the $\theta_{K}$ region 0.6 to 0.7 . These broad plots clearly show that obtaining highly polarized $\rho$ mesons does not require any fine-tuning nor very narrow angular selection. Self-polarization is an intrinsic, robust feature of this production scheme.

\section{EXPERIMENTAL PROSPECTS}

The present-day high-energy colliders are not designed to collide twisted particles. Novel instrumentation is needed to produce, accelerate, and focus particles in twisted states, which requires significant efforts from the beam and accelerator physics communities. Fortunately, the final state particles can be detected and analyzed with traditional detectors.

Despite these challenges, we believe that many of the phenomena proposed and studied in this paper can be verified with a modest experimental push, and we list here some steps toward achieving this goal:

(i) To remind the reader, twisted electrons with energies up to $300 \mathrm{keV}$, an excellent control over OAM, and focal spot stability have been produced [24]. Among several twisted electron produced schemes demonstrated so far, one is based on an artificial magnetic monopole, which effectively exists at the tip of a thin magnetized needle [41]. This method relies on electromagnetic interactions and can be applied to any charged particle, including positrons and (anti) protons. To realize it, one needs to prepare the initial charged particle in an approximately plane wave state with the transverse coherence length of the order of $1 \mu \mathrm{m}$. It remains to be seen if this can be achieved, for example, in experiments with trapped cold protons, antiprotons, or positrons.

(ii) Once a beam of low-energy electrons or other charged particles is prepared in a twisted state, it can be injected in a linear accelerator and accelerated to higher energies. This can be done either inside a conventional Radio Frequency (RF) cavity or in plasma wake field accelerators. In the ideal case of a 

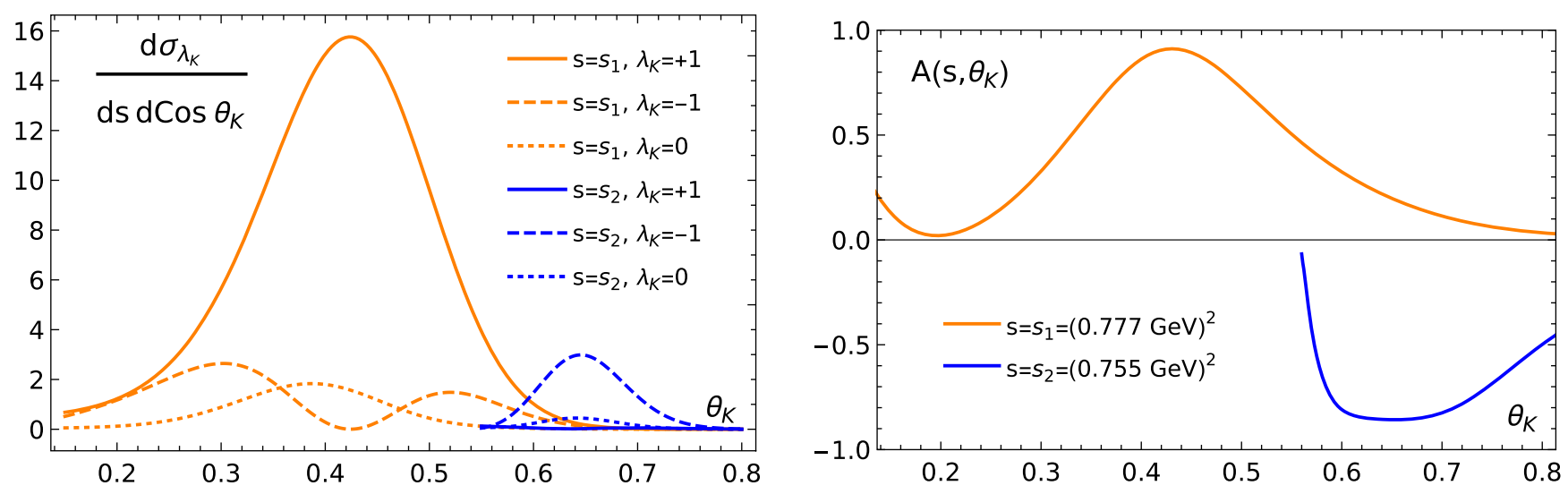

FIG. 9. Polarization properties of $\rho$ mesons produced in the unpolarized twisted $e^{+} e^{-}$annihilation with kinematic parameters as in Eq. (77). Left: Differential cross section (in arbitrary units) for the broad range of polar angles $0.1<\theta_{K}<0.8$ and for the two values of the center of mass energies: $\sqrt{s_{1}}=0.777 \mathrm{GeV}$ (lighter orange curves) and $\sqrt{s_{2}}=0.755 \mathrm{GeV}$ (darker blue curves). The solid, dashed, and short-dashed curves show the cross sections for $\lambda_{K}=+1,-1$, and 0 , respectively. Right: Differential polarization asymmetry $A$, Eq. (76), as a function of the polar angle $\theta_{K}$. The finite width effects are included according to Eq. (74).

perfectly longitudinal electric field and Bessel twisted state, the acceleration will boost the longitudinal momentum without affecting its transverse wave function. In a realistic situation, stray electromagnetic fields may be of concern as they may spoil the twisted state. We believe that the phase vortex of a twisted state, being topologically protected, will survive for sufficiently weak stray fields. This belief is further supported by the optical experiments with free space transmission of twisted light across the turbulent atmosphere over a distance of $143 \mathrm{~km}$ [42]. The exact tolerance levels can only be revealed by a dedicated numeric simulation and its experimental verification. These proof-of-principle studies are doable with the existing technologies.

(iii) Behavior of paraxial twisted electrons in storage rings was analytically studied in Refs. $[43,44]$, with the optimistic conclusions that orbital helicity evolves in a well-predicted way and can survive many rotation. Once again, these expectations must be corroborated by numerical simulations.

(iv) Once the twisted particles are accelerated and brought to collisions, one must be able to monitor whether their twisted state is indeed preserved and whether it is sufficiently stable. A suitable diagnostic tool is elastic scattering of twisted charged particles. This process was analyzed in Ref. [45], and characteristic observables were demonstrated.

(v) It is possible to bring twisted particles to the hadronic physics realm even without accelerating them. It suffices to bring slow protons and antiprotons to twisted states and arrange for their collisions $p \bar{p} \rightarrow$ hadrons. All features proposed in this paper will apply to this proof-of-principle experiment. The list of observables will be even larger due to the more complex final state, and its theoretical exploration is postponed to a future publication.

(vi) Finally, it is possible to arrange for an atomic physics counterpart of the experiments described here. Imagine a one-photon excitation of a trapped atom or ion, in which both initial particles are twisted: $\gamma_{\mathrm{tw}}+$ atom $_{\mathrm{tw}} \rightarrow$ atom*. All the kinematic and polarization effects discussed here will apply, albeit in different energy ranges. If one prefers to avoid dealing with twisted atoms, one can arrange for two-twisted-photon excitation of a trapped atom $\gamma_{1 \mathrm{tw}}+\gamma_{2 \mathrm{tw}}+$ atom $\rightarrow$ atom*, again keeping all the essential proposals of the present paper. We believe that such "twisted spectroscopy" is doable with the present-day technology. If successful, it will demonstrate the feasibility of the idea and open a new direction in optical spectroscopy.

\section{CONCLUSIONS}

Uncovering and exploring the spin properties of hadrons and their interactions is an intricate and fascinating topic in hadron phenomenology. There is a wealth of information revealed through spin-dependent observables in hadronic processes, but it is not always easy to extract and disentangle them experimentally. Although the spin physics program pursued by the hadronic community at specialized colliders is rich and multifaceted, any new complementary method of accessing spin observables would be very welcome.

In this work, we explored in detail the idea that we recently proposed in Ref. [12] that spin- and paritydependent observables can be studied even with unpolarized particles, provided they are prepared in twisted states. In such a state, particles possess a well-defined $z$ projection of the angular momentum, which receives contribution not 
only from spin but also from the orbital angular momentum. If one prepares a beam of photons or electrons with an equal amount of positive and negative helicities but with the angular momentum fixed, then the resonance production cross sections will display dramatic energy dependence and angular effects, which will reveal the spin-dependent observables in a novel way.

We showed how production of a hypothetical spin- 0 particle in collision of unpolarized twisted photons can reveal the amount of its scalar-pseudoscalar mixing. This is an illustration of the power of twisted particles in detecting parity-violating effects in the fully unpolarized case. We also demonstrated that vector mesons produced in unpolarized twisted $e^{+} e^{-}$annihilation can in fact be almost $100 \%$ polarized and their polarization state can be controlled by adjusting kinematics of the colliding twisted particles. None of these effects is possible with the usual plane wave collisions.

All these opportunities offer a remarkably rich pattern of observable spin physics effects which can be probed in twisted particle collisions, although not yet at existing colliders. Twisted electrons and photons have been experimentally demonstrated only for low energies, and one needs first to prepare high-energy twisted particles and bring them into collisions. This field is barely explored, but there exist theoretical suggestions such as in Refs. [13,14] which await exploration. We believe that the novel opportunities in hadronic physics offered by twisted particles present a sufficiently compelling scientific case to justify further dedicated work on their realization.

\section{ACKNOWLEDGMENTS}

I. P. I. thanks the Institute of Modern Physics, Lanzhou, China, for financial support and hospitality during his stay. I. P. I. acknowledges funding from the Portuguese Fundação para a Ciência e a Tecnologia (FCT) through the FCT Investigator Contract No. IF/00989/2014/CP1214/ CT0004 under the IF2014 program and through Contracts No. PTDC/FIS-PAR/29436/2017, No. UID/FIS/00777/ 2019, and No. CERN/FIS-PAR/0004/2017, which are partially funded through Programa Operacional Competitividade e Internacionalização (POCI), Quadro de Referência Estratégica Nacional (QREN), and the European Union. I. P. I. also acknowledges the support from National Science Center, Poland, via the project Harmonia (Project No. UMO-2015/18/M/ST2/00518). P. M.Z. and N. K. are supported by the National Natural Science Foundation of China via Grants No. 11975320 (P. M. Z.) and No. 11875296 (N. K.). A. V. P. and N. K. thank the Chinese Academy of Sciences President's International Fellowship Initiative for the support via Grants No. 2019PM0036 (A. V. P.) and No. 2020PM0073 (N. K.). The work has been partially supported by the Ministry of Education and Science of the Russian Federation: Projects No. 3.6371.2017/8.9 and No. 3.6439.2017/8.9.
[1] M. Tanabashi et al. (Particle Data Group), Phys. Rev. D 98, 030001 (2018).

[2] G. Eichmann, H. Sanchis-Alepuz, R. Williams, R. Alkofer, and C. S. Fischer, Prog. Part. Nucl. Phys. 91, 1 (2016).

[3] S. L. Olsen, Front. Phys. 10, 121 (2015).

[4] A. Pimikov, H.-J. Lee, and P. Zhang, Phys. Rev. D 101, 014002 (2020).

[5] A. Pimikov, H.-J. Lee, N. Kochelev, P. Zhang, and V. Khandramai, Phys. Rev. D 96, 114024 (2017).

[6] C. A. Aidala, S. D. Bass, D. Hasch, and G. K. Mallot, Rev. Mod. Phys. 85, 655 (2013).

[7] E. Leader and C. Lorcé, Phys. Rep. 541, 163 (2014).

[8] M. Anselmino, M. Boglione, U. D’Alesio, A. Kotzinian, F. Murgia, A. Prokudin, and C. Turk, Phys. Rev. D 75, 054032 (2007).

[9] M. Anselmino, A. Mukherjee, and A. Vossen, arXiv: 2001.05415.

[10] D. Boer, Proc. Sci., SPIN2018 (2019) 167 [arXiv: 1903.01119].

[11] X. Chen, Proc. Sci., DIS2018 (2018) 170 [arXiv: 1809.00448].

[12] I. P. Ivanov, N. Korchagin, A. Pimikov, and P. M. Zhang, Phys. Rev. Lett. 124, 192001 (2020).
[13] U. D. Jentschura and V. G. Serbo, Phys. Rev. Lett. 106, 013001 (2011).

[14] U. D. Jentschura and V. G. Serbo, Eur. Phys. J. C 71, 1571 (2011).

[15] G. L. Kotkin, V. G. Serbo, and A. Schiller, Int. J. Mod. Phys. A 07, 4707 (1992).

[16] I. P. Ivanov, Phys. Rev. D 83, 093001 (2011).

[17] I. P. Ivanov and V. G. Serbo, Phys. Rev. A 84, 033804 (2011).

[18] D. V. Karlovets, Phys. Rev. A 86, 062102 (2012).

[19] D. V. Karlovets, G. L. Kotkin, and V. G. Serbo, Phys. Rev. A 92, 052703 (2015).

[20] D. Karlovets, J. High Energy Phys. 03 (2017) 049.

[21] D. Karlovets, Phys. Rev. A 98, 012137 (2018).

[22] A. J. Silenko, P. Zhang, and L. Zou, Phys. Rev. A 100, 030101(R) (2019).

[23] D. V. Karlovets and V. G. Serbo, Phys. Rev. D 101, 076009 (2020).

[24] K. Y. Bliokh et al., Phys. Rep. 690, 1 (2017).

[25] S. M. Lloyd, M. Babiker, G. Thirunavukkarasu, and J. Yuan, Rev. Mod. Phys. 89, 035004 (2017).

[26] M. J. Padgett, Opt. Express 25, 11265 (2017).

[27] B. A. Knyazev and V. G. Serbo, Phys. Usp. 61, 449 (2018). 
[28] V. Serbo, I. P. Ivanov, S. Fritzsche, D. Seipt, and A. Surzhykov, Phys. Rev. A 92, 012705 (2015).

[29] L. D. Landau and E. M. Lifshits, Quantum Mechanics, Course of Theoretical Physics Vol. 3 (Butterworth-Heinemann, Oxford, 1991).

[30] K. Y. Bliokh, F. J. Rodríguez-Fortuño, F. Nori, and A. V. Zayats, Nat. Photonics 9, 796 (2015).

[31] M. Uchida and A. Tonomura, Nature (London) 464, 737 (2010).

[32] J. Verbeeck, H. Tian, and P. Schattschneider, Nature (London) 467, 301 (2010).

[33] B. J. McMorran, A. Agrawal, I. M. Anderson, A. A. Herzing, H. J. Lezec, J. J. McClelland, and J. Unguris, Science 331, 192 (2011).

[34] K. Y. Bliokh, M. R. Dennis, and F. Nori, Phys. Rev. Lett. 107, 174802 (2011).

[35] Y. Zhao, J. S. Edgar, G. D. M. Jeffries, D. McGloin, and D. T. Chiu, Phys. Rev. Lett. 99, 073901 (2007).

[36] I. P. Ivanov, N. Korchagin, A. Pimikov, and P. Zhang, Phys. Rev. D 101, 016007 (2020).
[37] V. B. Berestetskii, E. M. Lifshitz, and L. P. Pitaevskii, Quantum Electrodynamics, Course of Theoretical Physics Vol. 4 (Pergamon Press, Oxford, 1982).

[38] I. P. Ivanov, D. Seipt, A. Surzhykov, and S. Fritzsche, Europhys. Lett. 115, 41001 (2016).

[39] I. P. Ivanov, V. G. Serbo, and P. Zhang, J. Opt. 21, 114001 (2019).

[40] I. P. Ivanov and N. N. Nikolaev, JETP Lett. 69, 294 (1999).

[41] A. Béché, R. Van Boxem, G. Van Tendeloo, and J. Verbeeck, Nat. Phys. 10, 26 (2014).

[42] M. Krenn, J. Handsteiner, M. Fink, R. Fickler, R. Ursin, M. Malik, and A. Zeilinger, Proc. Natl. Acad. Sci. U.S.A. 113, 13648 (2016).

[43] G. M. Gallatin and B. McMorran, Phys. Rev. A 86, 012701 (2012).

[44] A. J. Silenko and O. V. Teryaev, Phys. Part. Nucl. Lett. 16, 77 (2019).

[45] I. P. Ivanov, D. Seipt, A. Surzhykov, and S. Fritzsche, Phys. Rev. D 94, 076001 (2016). 